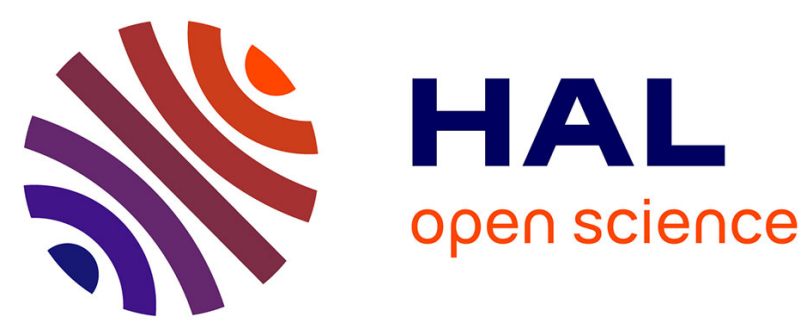

\title{
Equivalent transmission conditions for the time-harmonic Maxwell equations in 3D for a medium with a highly conductive thin sheet
}

Victor Péron, Kersten Schmidt, Marc Duruflé

\section{- To cite this version:}

Victor Péron, Kersten Schmidt, Marc Duruflé. Equivalent transmission conditions for the timeharmonic Maxwell equations in 3D for a medium with a highly conductive thin sheet. SIAM Journal on Applied Mathematics, 2016, 76 (3), pp.1031-1052. 10.1137/15M1012116 . hal-01260111v2

HAL Id: hal-01260111

https://hal.science/hal-01260111v2

Submitted on 2 Apr 2016

HAL is a multi-disciplinary open access archive for the deposit and dissemination of scientific research documents, whether they are published or not. The documents may come from teaching and research institutions in France or abroad, or from public or private research centers.
L'archive ouverte pluridisciplinaire HAL, est destinée au dépôt et à la diffusion de documents scientifiques de niveau recherche, publiés ou non, émanant des établissements d'enseignement et de recherche français ou étrangers, des laboratoires publics ou privés.

\section{(c)(1)}

Distributed under a Creative Commons Attribution| 4.0 International License 


\title{
EQUIVALENT TRANSMISSION CONDITIONS FOR THE TIME-HARMONIC MAXWELL EQUATIONS IN 3D FOR A MEDIUM WITH A HIGHLY CONDUCTIVE THIN SHEET
}

\author{
V. PÉRON* ${ }^{*}$ K. SCHMIDT ${ }^{\dagger}$, AND M. DURUFLÉ
}

\begin{abstract}
We propose equivalent transmission conditions of order 1 and 2 for thin and highly conducting sheets for the time-harmonic Maxwell's equation in three dimension. The transmission conditions are derived asymptotically for vanishing sheet thickness $\varepsilon$ where the skin depth is kept proportional to $\varepsilon$. The condition of order 1 turns out to be the perfect electric conductor boundary condition. The conditions of order 2 appear as generalised Poincaré-Steklov maps between tangential components of the magnetic field and the electric field, and they are of Wentzell type involving second order surface differential operators. Numerical results with finite elements of higher order validate the asymptotic convergence for $\varepsilon \rightarrow 0$ and the robustness of the equivalent transmission condition of order 2 .
\end{abstract}

Key words. Impedance Transmission Conditions, Thin Conducting Sheets, Asymptotic Expansions, Time-harmonic Maxwell's equations

AMS subject classifications. 35C20, 35J25, 41A60,

1. Introduction. Many electric and electronic devices feature thin conducting sheets providing efficient electromagnetic shielding. Due to their large aspect ratio and high conductivity of the flat or curved sheets the shielding properties are achieved with a minimum use of materials. Precisely their large aspect ratio makes the numerical simulation of such devices more of a challenge, especially if standard methods like the finite element method (FEM) or the finite difference method shall be applied. As the sheets have to be solved by the mesh in thickness direction, the number of cells in the mesh increase with decreasing sheet thickness. In addition for higher frequencies, the fields decay rapidly inside the sheets and can be only resolved by increasing the number of cells even more. The numerical modelling is much simplified if the thin conducting sheets are replaced by transmission conditions on an interface, which is usually its mid-surface. Using the so called impedance transmission conditions, which relate the electric and magnetic fields on both sides of the interface, meshes with much larger cells can be used. Providing an accurate prediction of the electromagnetic fields, those transmission conditions are called equivalent.

Already in 1902 Levi-Civita introduced equivalent transmission conditions [17] (see also [1,33]) for Maxwell's equations. He postulated that the electric field is continuous over the interface whereas the magnetic field has a discontinuity, which is proportional to the sheet thickness and conductivity. These conditions fit naturally with boundary integral formulations $[15,20]$ as well as finite element methods $[25,26$, $2,14]$. Schmidt and Tordeux [31] have shown in 2010 for the eddy current model in 2D that these conditions, which they call ITC-1-0, appear as the asymptotic limit when the sheet thickness $\varepsilon$ tends to zero while the conductivity tends to infinity like $1 / \varepsilon$. In this case it has been shown [28] that these transmission conditions exhibit a robust linear error reduction with $\varepsilon$, i.e., independent of the conductivity or frequency. As

* LMAP CNRS UMR 5142 \& Team MAGIQUE 3D INRIA, Université de Pau et des Pays de l'Adour, 64013 Pau, France (victor.peron@inria.fr)

${ }^{\dagger}$ Research center MATHEON and Institut für Mathematik, Technische Universität Berlin, 10623 Berlin, Germany (kersten.schmidt@math.tu-berlin.de).

${ }^{\ddagger}$ Team Magique-3D, INRIA Bordeaux-Sud-Ouest, Institut de Mathématiques de Bordeaux, CNRS UMR 5251 \& Université de Bordeaux1, 33405 Talence Cedex, France (marc.durufle@inria.fr) 
variation of the conditions by Levi-Civita the so called shielding element [22] has been introduced. Recently, conditions in the framework of Schmidt and Tordeux have been derived for the axis-symmetric setting and varying thickness [11].

In an alternative way the so called thin layer boundary condition $[34,16,18,13,10]$ are derived taking into account the boundary layer behaviour of the solution. More precisely, the fields are for higher conductivities or frequencies mainly hyberbolic functions inside the sheet. In this way, the thin layer boundary conditions exhibit jumps in both the electric and magnetic field and involve sheet thickness and conductivity as arguments of hyberbolic functions. The thin layer boundary conditions come with an increased complexity, but it has been shown that they do not lead to lower error levels than the simpler ITC-1-0 conditions [28].

Equivalent transmission conditions with drastically reduced error levels can systematically been derived by an asymptotic analysis of the Maxwell's equations with thin conducting sheets where the sheet thickness $\varepsilon$ tends to zero. For example, for the eddy current model in 2D two families of transmission conditions have been derived using asymptotic expansions, this is the family ITC-1-N [32] in which the conductivity is scaled with the sheet thickness $\varepsilon$ like $1 / \varepsilon$, and the family ITC-2-N [27, 29], in which the conductivity is scaled like $1 / \varepsilon^{2}$. For both families $N$ corresponds to the order, where the convergence of the modelling error outside the sheet is like $\varepsilon^{N+1}$ in their respective asymptotic regime. However, this convergence is not always robust in terms of the conductivity. For instance, in [28] it has been shown that the ITC-1-1 conditions increase the accuracy in comparison to the ITC-1-0 conditions and the ITC-1-2 conditions in comparison to the ITC-1-1 conditions if the sheet conductivity or frequency are not too high. Otherwise, the accuracy remains the same or is even reduced when the order of the transmission conditions is increased. It turns out that the ITC-2-0 and ITC-2-1 transmission conditions are robust and can be used from very low to very high frequencies. For the ITC-2-1 conditions a quadratic convergence of the modelling in the sheet thickness has been observed numerically, which is uniform in the conductivity or frequency. The many different equivalent transmission conditions in two dimensions can be easily integrated in finite element methods or boundary element methods [30], where for the latter only the interface has to be discretized.

Since most electromagnetic devices necessitate the modelling in three dimensions we aim to derive equivalent transmission conditions for the full time-harmonic Maxwell's equations in 3D and to investigate numerically their properties. To obtain robust transmission condition as the ITC-2-1 in two dimensions we choose the asymptotic regime in which the conductivity is scaled like $1 / \varepsilon^{2}$. We consider the general case of curved thin sheets where all the material constants may take different values inside and on the two sides of the sheet. Here, we use techniques which have been used in a similar way to derive transmission conditions for other electromagnetic models in 3 D including thin layers $[8,7,9]$. In this paper we restrict ourselves to the derivation and numerical verification of the transmission conditions. The main tools (Helmholtz decomposition) for a theoretical justification can be found in $[5,8,7,9]$.

The paper is organized as follows. Section 2 presents the model with a formulation in terms of the electric field and a formal derivation of equivalent conditions based on an asymptotic expansion in the thickness parameter $\varepsilon$. Then, in Section 3 as main results the equivalent model of order 1, which satisfies PEC boundary conditions, and the equivalent model of order 2 are given, and compared to the Levi-Civita and the thin layer boundary conditions. The derivation of the equivalent models is 
based on an asymptotic expansion which is presented in detail and order by order in Section 5. Further details are given in Appendix A. The equivalent model of order 2 involves as a transmission condition a generalised Poincaré-Steklov map (tangential components of magnetic field to tangential components of electric field) with a second order surface differential operator, whose structure simplifies for a symmetric configuration of material constants. We introduce at the end of the Section 3 a mixed variational formulation including as additional unknowns the mean and jump of the tangential magnetic field components on the interface. The properties of the equivalent models are studied numerically in Section 4 for two examples with both a general non-symmetric setting of the material properties, the shielding of an incoming plane wave by a spherical thin conducting sheet and the shielding of a Gaussian current source by a flat conducting layer. These studies include the convergence of the modelling error and the robustness with respect to the sheet conductivity.

2. The mathematical model and equivalent models with transmission conditions. After the introduction of notations in Sec. 2.1 and the mathematical model for the electric and magnetic field in Sec. 2.2 we infer the electric field formulation in Sec. 2.3. Then we present a guideline on the derivation of equivalent conditions (section 2.4), where equivalent conditions for the considered model will be given up to order 2 in the Sec. 3.

2.1. Notations. For any orientable and closed surface $\Gamma$ of $\mathbb{R}^{3}$ the unit normal vector $\mathbf{n}$ on $\Gamma$ is outwardly oriented from the interior domain enclosed by $\Gamma$ towards the outer domain, see e.g. Fig. 2.1. Let $\mathbf{v}$ a vector field on $\Gamma$, then we denote by

$$
\mathbf{v}_{\mathbf{T}}=\mathbf{n} \times(\mathbf{v} \times \mathbf{n}),
$$

the vector field of its tangent components and the space of $L^{2}$-integrable tangent vector fields by $\mathbf{L}_{t}^{2}(\Gamma):=\left\{\mathbf{v} \in\left(\mathrm{L}^{2}(\Gamma)\right)^{3}, \mathbf{v} \cdot \mathbf{n}=0\right.$ on $\left.\Gamma\right\}$.

We denote by $\operatorname{curl}_{\Gamma}$ the tangential rotational operator (which applies to functions defined on $\Gamma$ ) and by $\operatorname{curl}_{\Gamma}$ the surface rotational operator (which applies to vector fields) $[23,9]$ :

$$
\begin{aligned}
\forall f \in C^{\infty}(\Gamma), \quad \operatorname{curl}_{\Gamma} f & =\left(\nabla_{\Gamma} f\right) \times \mathbf{n}, \\
\forall \mathbf{v} \in\left(C^{\infty}(\Gamma)\right)^{3}, & \operatorname{curl}_{\Gamma} \mathbf{v}=\operatorname{div}_{\Gamma}(\mathbf{v} \times \mathbf{n}),
\end{aligned}
$$

where $\nabla_{\Gamma}$ and $\operatorname{div}_{\Gamma}$ are respectively the tangential gradient and the surface divergence on $\Gamma$. This allows us to define the space of tangent vector fields of the operator $\operatorname{curl}_{\Gamma}[23,9]$ :

$$
\operatorname{TH}\left(\operatorname{curl}_{\Gamma}, \Gamma\right)=\left\{\mathbf{v} \in \mathbf{L}_{t}^{2}(\Gamma), \operatorname{curl}_{\Gamma} \mathbf{v} \in \mathrm{L}^{2}(\Gamma)\right\},
$$

which is, equipped with the graph norm of $\operatorname{curl}_{\Gamma}$, a Hilbert space.

Let $\Omega_{-}$and $\Omega_{+}$be Lipschitz domains with a common interface $\Gamma:=\partial \Omega_{-} \cap \partial \Omega_{+}$, which is a closed set, and let $\mathbf{n}$ on $\Gamma$ be the unit normal vector directed into $\Omega_{+}$(see Fig. 2.2). Then, for functions $f \in C^{\infty}\left(\Omega_{ \pm}\right)$, which are possibly discontinuous over the interface $\Gamma$, we denote by $[f]_{\Gamma}$ the jump of $f$ across $\Gamma$ :

$$
[f]_{\Gamma}=\left.f\right|_{\Gamma^{+}}-\left.f\right|_{\Gamma^{-}}
$$

where for any $\mathbf{x}_{\Gamma} \in \Gamma$ the one-sided traces are defined by

$$
\left.f\right|_{\Gamma^{ \pm}}\left(\mathbf{x}_{\Gamma}\right):=\lim _{s \rightarrow 0^{ \pm}} f\left(\mathbf{x}_{\Gamma}+s \mathbf{n}\right) .
$$


Furthermore, we denote by $\{f\}_{\Gamma}$ the mean value of $f$ across $\Gamma$ :

$$
\{f\}_{\Gamma}=\frac{1}{2}\left(\left.f\right|_{\Gamma^{+}}+\left.f\right|_{\Gamma^{-}}\right) .
$$

We use the same definition for vector fields $\mathbf{v} \in\left(C^{\infty}\left(\Omega_{ \pm}\right)\right)^{3}$, and with an abuse of notation, for the tangential traces:

$$
\begin{array}{rlrl}
\{\mathbf{v} \times \mathbf{n}\}_{\Gamma} & :=\{\mathbf{v}\}_{\Gamma} \times \mathbf{n}, & {[\mathbf{v} \times \mathbf{n}]_{\Gamma}:} & =[\mathbf{v}]_{\Gamma} \times \mathbf{n}, \\
\left\{\mathbf{v}_{\mathbf{T}}\right\}_{\Gamma}: & =\left(\{\mathbf{v}\}_{\Gamma}\right)_{\mathbf{T}}, & {\left[\mathbf{v}_{\mathbf{T}}\right]_{\Gamma}:=\left([\mathbf{v}]_{\Gamma}\right)_{\mathbf{T}} .}
\end{array}
$$

Finally, we define by $\mathbf{H}\left(\operatorname{curl}, \Omega_{ \pm}\right)$the completion of the space $\left(C^{\infty}\left(\bar{\Omega}_{ \pm}\right)\right)^{3}$ with respect to the natural graph norm of curl, which is a Hilbert space as well. Then, for vector fields $\mathbf{v} \in \mathbf{H}\left(\operatorname{curl}, \Omega_{ \pm}\right)$both the jump $[\mathbf{v} \times \mathbf{n}]_{\Gamma}$ and the mean value $\{\mathbf{v} \times \mathbf{n}\}_{\Gamma}$ are in the Hilbert space

$$
\mathrm{TH}^{-1 / 2}\left(\operatorname{div}_{\Gamma}, \Gamma\right):=\left\{\mathbf{v} \in\left(H^{-1 / 2}(\Gamma)\right)^{3}, \operatorname{div}_{\Gamma} \mathbf{v} \in \mathrm{H}^{-1 / 2}(\Gamma)\right\}
$$

which is, equipped with the graph norm $\left(\|\mathbf{v}\|_{H^{-1 / 2}(\Gamma)}^{2}+\left\|\operatorname{div}_{\Gamma} \mathbf{v}\right\|_{H^{-1 / 2}(\Gamma)}^{2}\right)^{1 / 2}$ of the operator $\operatorname{div}_{\Gamma}$, the dual of $\operatorname{TH}\left(\operatorname{curl}_{\Gamma}, \Gamma\right)$.

2.2. Time-harmonic Maxwell equations. Throughout the paper we denote by $\Omega \subset \mathbb{R}^{3}$ the domain of interest, which is composed of three subdomains (see Figure 2.1) as

$$
\Omega=\Omega_{-}^{\varepsilon} \cup \overline{\Omega_{o}^{\varepsilon}} \cup \Omega_{+}^{\varepsilon}
$$

corresponding to different linear materials. The subdomain $\Omega_{o}^{\varepsilon}$ is a thin layer of constant thickness $\varepsilon$ surrounding the subdomain $\Omega_{-}^{\varepsilon}$. The boundary of the subdomain $\Omega_{-}^{\varepsilon}$ is the smooth surface denoted by $\Gamma_{-}^{\varepsilon}$ while $\Gamma_{+}^{\varepsilon}$ is the boundary of the subdomain $\overline{\Omega_{-}^{\varepsilon}} \cup \Omega_{o}^{\varepsilon}$. The mid-surface of the thin layer $\Omega_{o}^{\varepsilon}$ is denoted by $\Gamma$.

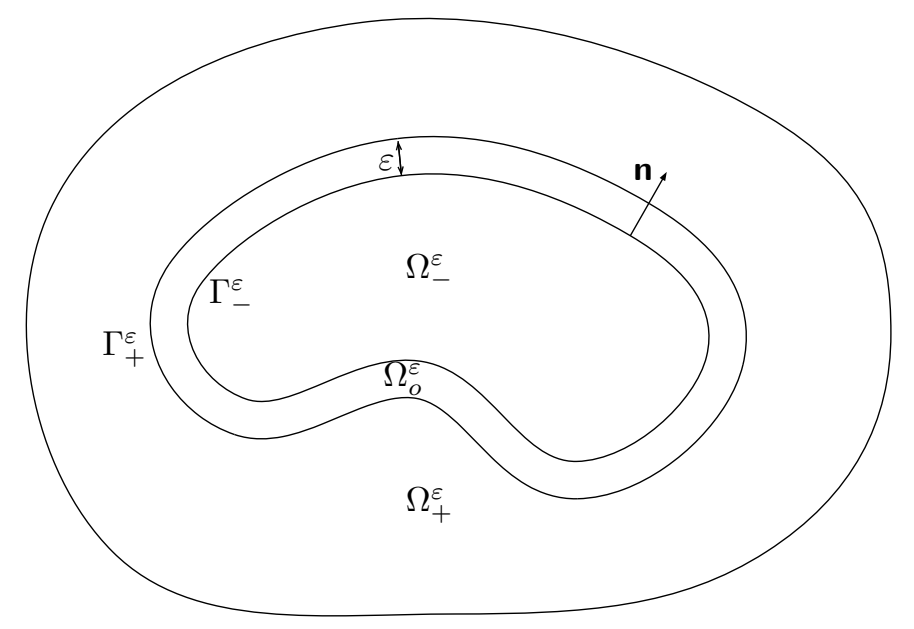

FIG. 2.1. A cross-section of the domain $\Omega$ and the subdomains $\Omega_{-}^{\varepsilon}, \Omega_{o}^{\varepsilon}, \Omega_{+}^{\varepsilon}$. 
The electromagnetic properties in $\Omega$ are given by the piecewise-constant functions $\mu^{\varepsilon}, \underline{\epsilon}^{\varepsilon}$, and $\underline{\sigma}^{\varepsilon}$ corresponding to the respective magnetic permeability, electric permittivity, and conductivity of the possibly different materials in the three subdomains. They are given by

$$
\underline{\mu}^{\varepsilon}=\left\{\begin{array}{ll}
\mu_{-}, & \text {in } \Omega_{-}^{\varepsilon}, \\
\mu_{o}, & \text { in } \Omega_{o}^{\varepsilon}, \\
\mu_{+}, & \text {in } \Omega_{+}^{\varepsilon},
\end{array} \quad \underline{\epsilon}^{\varepsilon}=\left\{\begin{array}{ll}
\epsilon_{-}, & \text {in } \Omega_{-}^{\varepsilon}, \\
\epsilon_{o}, & \text { in } \Omega_{o}^{\varepsilon}, \\
\epsilon_{+}, & \text {in } \Omega_{+}^{\varepsilon},
\end{array} \quad \underline{\sigma}^{\varepsilon}= \begin{cases}\sigma_{-}, & \text {in } \Omega_{-}^{\varepsilon}, \\
\sigma_{o}^{\varepsilon}=\varepsilon^{-2} \tilde{\sigma}, & \text { in } \Omega_{o}^{\varepsilon}, \\
\sigma_{+}, & \text {in } \Omega_{+}^{\varepsilon} .\end{cases}\right.\right.
$$

We consider $\varepsilon$ as a parameter, on which $\underline{\mu}^{\varepsilon}$ and $\underline{\epsilon}^{\varepsilon}$ depend through the definition of the subdomains, where in $\underline{\sigma}^{\varepsilon}$ in addition we assume an explicit dependence of the layer conductivity $\sigma_{o}^{\varepsilon}$ on $\varepsilon$. With this correlation the thinner is the layer, the larger is the conductivity in the layer. The dependence like $\varepsilon^{-2}$ corresponds for $\varepsilon \rightarrow 0$ to asymptotically constant ratio of skin depth $d_{\text {skin }}=\sqrt{2 /\left(\omega \mu_{o} \sigma_{o}^{\varepsilon}\right)}$ and thickness $\varepsilon$ [28], i.e., they behave the same for $\varepsilon \rightarrow 0$.

Let us denote by $\mathbf{j}$ the time-harmonic current source (with time convention $\exp (-\mathrm{i} \omega t)$ ) and let $\omega>0$ be the angular frequency. For the sake of simplicity, we assume that $\mathbf{j}$ is smooth enough and the support of $\mathbf{j}$ does not meet the layer $\Omega_{o}^{\varepsilon}$, and we write $\mathbf{j}_{ \pm}=\mathbf{j}$ in $\Omega_{ \pm}^{\varepsilon}$. Maxwell's equations link the electric field $\mathbf{E}$ and the magnetic field $\mathbf{H}$, through Faraday's and Ampère's laws in $\Omega$ :

$$
\operatorname{curl} \mathbf{E}^{\varepsilon}-i \omega \underline{\mu}^{\varepsilon} \mathbf{H}^{\varepsilon}=0 \text { and } \operatorname{curl} \mathbf{H}^{\varepsilon}+\left(i \omega \underline{\epsilon}^{\varepsilon}-\underline{\sigma}^{\varepsilon}\right) \mathbf{E}^{\varepsilon}=\mathbf{j} \text { in } \Omega \text {. }
$$

We complement this problem with a Silver-Müller boundary condition [21] set on $\partial \Omega$.

2.3. Electric field formulation. We denote by $\underline{\kappa}^{\varepsilon}$ the complex wave number given by

$$
\left(\underline{\kappa}^{\varepsilon}\right)^{2}(x)=\omega^{2} \underline{\mu}^{\varepsilon}(x)\left(\underline{\epsilon}^{\varepsilon}(x)+\mathrm{i} \frac{\underline{\sigma}^{\varepsilon}(x)}{\omega}\right), \quad \operatorname{Im}\left(\underline{\kappa}^{\varepsilon}(x)\right) \geq 0 .
$$

In the framework above, $\underline{\kappa}^{\varepsilon}$ is a piecewise-constant function defined inside the three subdomains as

$$
\underline{\kappa}^{\varepsilon}= \begin{cases}\kappa_{-}, & \text {in } \Omega_{-}^{\varepsilon}, \\ \kappa_{o}^{\varepsilon}, & \text { in } \Omega_{o}^{\varepsilon} \\ \kappa_{+}, & \text {in } \Omega_{+}^{\varepsilon} .\end{cases}
$$

Then, Maxwell's system of first order partial differential equations can be reduced to the following second-order equation for the electric field

$$
\operatorname{curl} \operatorname{curl} \mathbf{E}^{\varepsilon}-\left(\underline{\kappa}^{\varepsilon}\right)^{2} \mathbf{E}^{\varepsilon}=i \omega \underline{\mu \mathbf{j}}, \quad \text { in } \quad \Omega_{-}^{\varepsilon} \cup \Omega_{o}^{\varepsilon} \cup \Omega_{+}^{\varepsilon},
$$

with the continuity conditions for the Dirichlet and Neumann traces ( $c f . \quad[12$, Sec. 3]) across the two conductor surfaces $\Gamma_{+}^{\varepsilon}$ and $\Gamma_{-}^{\varepsilon}$

$$
\begin{aligned}
& \mathbf{E}_{ \pm}^{\varepsilon} \times \mathbf{n}=\mathbf{E}_{o}^{\varepsilon} \times \mathbf{n}, \quad \text { on } \quad \Gamma_{ \pm}^{\varepsilon}, \\
& \frac{1}{\mu_{ \pm}} \operatorname{curl} \mathbf{E}_{ \pm}^{\varepsilon} \times \mathbf{n}=\frac{1}{\mu_{o}} \operatorname{curl} \mathbf{E}_{o}^{\varepsilon} \times \mathbf{n}, \quad \text { on } \Gamma_{ \pm}^{\varepsilon},
\end{aligned}
$$

and with the boundary conditions

$$
\operatorname{curl} \mathbf{E}_{+}^{\varepsilon} \times \mathbf{n}-i \kappa_{+} \mathbf{n} \times \mathbf{E}_{+}^{\varepsilon} \times \mathbf{n}=0 \quad \text { on } \partial \Omega .
$$

Here $\mathbf{E}_{\dagger}^{\varepsilon}, \dagger=-, o,+$ denote the restrictions of $\mathbf{E}^{\varepsilon}$ to the respective subdomain $\Omega_{\dagger}^{\varepsilon}$. 
2.4. Guideline on the derivation of equivalent conditions. Discretizing the model (2.1) with the conducting sheet by the finite element method or any other standard discretization technique the layer has to be resolved by the mesh. If the layer is thin and, if, moreover, the electric fields decay rapidly inside the layer due to high conductivities (the skin effect), then meshes with very small cells are required. The meshing of the thin layer can be avoided if it is replaced by an interface, usually its mid-surface $\Gamma$, on which appropriate conditions are set.

We give a guideline on the derivation of equivalent conditions in this section, which is based on an asymptotic expansion in the thickness parameter $\varepsilon$ in Sect. 5 . We will then propose two equivalent models $\mathbf{E}_{0}$ in Sec. 3.1 and $\mathbf{E}_{\varepsilon}^{1}$ in Sec. 3.2, both for the electric field. The first model $\mathbf{E}_{0}$ is of order 1, i.e., it satisfies at least formally $\mathbf{E}^{\varepsilon}-\mathbf{E}_{0}=\mathcal{O}(\varepsilon)$ and the second model $\mathbf{E}_{\varepsilon}^{1}$ is of order 2, i.e. it satisfies at least formally $\mathbf{E}^{\varepsilon}-\mathbf{E}_{\varepsilon}^{1}=\mathcal{O}\left(\varepsilon^{2}\right)$. These models are defined in $\varepsilon$-independent domains $\Omega_{-}, \Omega_{+}$, where $\Omega_{-}$denotes the domain $\Omega_{-}^{\varepsilon}$ in the limit $\varepsilon \rightarrow 0$ and $\Omega_{+}$the domain $\Omega_{+}^{\varepsilon}$ for $\varepsilon \rightarrow 0$, i.e. $\Omega_{+}=\Omega \backslash \bar{\Omega}_{-}$(see Figure 2.2).

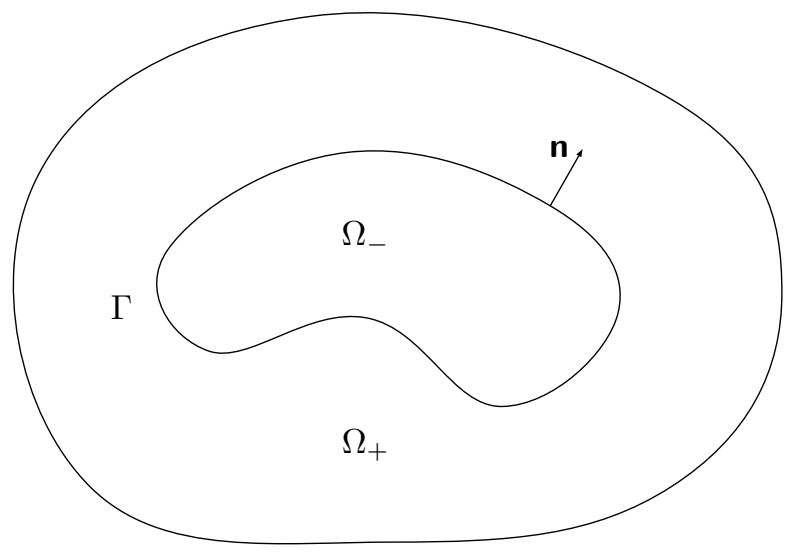

FIG. 2.2. A cross-section of the domain $\Omega$ and the subdomains $\Omega_{-}, \Omega_{+}$.

To define these equivalent models it is convenient to introduce the electromagnetic properties of the "background" problem by simple extension of the values of $\underline{\mu}^{\varepsilon}, \underline{\epsilon}^{\varepsilon}$ and $\underline{\sigma}^{\varepsilon}$ outside the sheet in the extended domains $\Omega_{-}$and $\Omega_{+}$:

$$
\mu=\left\{\begin{array}{ll}
\mu_{-}, & \text {in } \Omega_{-}, \\
\mu_{+}, & \text {in } \Omega_{+},
\end{array} \quad \epsilon=\left\{\begin{array}{ll}
\epsilon_{-}, & \text {in } \Omega_{-}, \\
\epsilon_{+}, & \text {in } \Omega_{+},
\end{array} \quad \sigma= \begin{cases}\sigma_{-}, & \text {in } \Omega_{-}, \\
\sigma_{+}, & \text {in } \Omega_{+} .\end{cases}\right.\right.
$$

Similarly, we define a complex wave number $\kappa$ as

$$
\kappa= \begin{cases}\kappa_{-}, & \text {in } \Omega_{-}, \\ \kappa_{+}, & \text {in } \Omega_{+}\end{cases}
$$

In the following we present briefly a formal derivation of equivalent conditions. We summarize this process in two steps.

First step : a multiscale expansion. The first step consists in deriving a multiscale expansion for the solution $\mathbf{E}^{\varepsilon}$ of the model problem (2.1) : it possesses an 
asymptotic expansion in power series of the small parameter $\varepsilon$

$$
\begin{array}{ll}
\mathbf{E}^{\varepsilon}(\mathbf{x}) \approx \mathbf{E}_{0}(\mathbf{x})+\varepsilon \mathbf{E}_{1}(\mathbf{x})+\varepsilon^{2} \mathbf{E}_{2}(\mathbf{x})+\cdots & \text { for a.e. } \quad \mathbf{x} \in \Omega_{-}^{\varepsilon} \cup \Omega_{+}^{\varepsilon}, \\
\mathbf{E}^{\varepsilon}(\mathbf{x}) \approx \mathfrak{E}_{0}\left(y_{\alpha}, \frac{h}{\varepsilon}\right)+\varepsilon \mathfrak{E}_{1}\left(y_{\alpha}, \frac{h}{\varepsilon}\right)+\cdots & \text { for a.e. } \quad \mathbf{x} \in \Omega_{o}^{\varepsilon} .
\end{array}
$$

Here, $\mathbf{x} \in \mathbb{R}^{3}$ are the cartesian coordinates, $\left(y_{\alpha}, h\right)$ is a local normal coordinate system [4] to the surface $\Gamma$ in the thin layer $\Omega_{o}^{\varepsilon}$ where $y_{\alpha}, \alpha=1,2$ are tangential coordinates on $\Gamma$ and $h \in\left(-\frac{\varepsilon}{2}, \frac{\varepsilon}{2}\right)$ is the normal coordinate to $\Gamma$. Moreover, the term $\mathfrak{E}_{j}$ is a "profile" defined on $\Gamma \times\left(-\frac{1}{2}, \frac{1}{2}\right)$. Note, that the intrinsic domain of the "far field terms" $\mathbf{E}_{j}$ is $\Omega_{-} \cup \Omega_{+}$. The first terms $\left(\mathfrak{E}_{j}, \mathbf{E}_{j}\right)$ for $j=0,1$ are formally derived step by step in Section 5 .

The derivation is based on an expansion of the differential operators inside the thin layer $\Omega_{o}^{\varepsilon}$ in terms of $\varepsilon$, a Taylor expansion of $\left.\mathbf{E}_{j}\right|_{\Gamma_{ \pm}^{\varepsilon}}$ around the mid-surface $\Gamma$ and a collection of terms of same powers of $\varepsilon$ in the governing PDE inside and outside the sheet, the continuity conditions for the Dirichlet and Neumann traces on $\Gamma_{ \pm}^{\varepsilon}$ and the boundary conditions. Since, moreover, the terms $\mathfrak{E}_{j}$ of the expansion inside the sheet can be explicitly expressed in terms of $\mathbf{E}_{i}, i=0, \ldots, j-1$ we obtain formally

$$
\begin{aligned}
\operatorname{curl} \operatorname{curl} \mathbf{E}_{j}^{ \pm}-\kappa^{2} \mathbf{E}_{j}^{ \pm} & =i \omega \mu \mathbf{j} \delta_{0 j}, & & \text { in } \Omega_{ \pm}, \\
\operatorname{curl} \mathbf{E}_{j}^{+} \times \mathbf{n}-i \kappa_{+} \mathbf{n} \times \mathbf{E}_{j}^{+} \times \mathbf{n} & =0, & & \text { on } \partial \Omega,
\end{aligned}
$$

for the restrictions $\mathbf{E}_{j}^{ \pm}$of $\mathbf{E}_{j}$ to $\Omega_{ \pm}$, with Dirichlet boundary conditions on $\Gamma$ :

$$
\mathbf{E}_{j}^{ \pm} \times \mathbf{n}=\sum_{i=0}^{j} \mathcal{G}_{i}^{ \pm}\left(\begin{array}{c}
\left\{\frac{1}{\mu}\left(\operatorname{curl} \mathbf{E}_{j-i}\right)_{\mathbf{T}}\right\}_{\Gamma} \\
{\left[\frac{1}{\mu}\left(\operatorname{corl} \mathbf{E}_{j-i}\right)_{\mathbf{T}}\right]_{\Gamma}}
\end{array}\right)
$$

Here, $\delta_{0 j}=1$ if $j=0$ and zero otherwise and $\mathcal{G}_{i}^{ \pm}$in the discrete convolution on the right hand side of $(2.3 \mathrm{c})$ are differential operators on $\Gamma$ not depending on $\varepsilon$ where $\mathcal{G}_{0}^{ \pm}=0$.

Second step : construction of equivalent conditions and equivalent models. The second step consists in identifying a simpler problem satisfied by an approximation $\mathbf{E}_{\varepsilon}^{k}$ of the truncated expansion $\mathbf{E}_{0}(\mathbf{x})+\varepsilon \mathbf{E}_{1}(\mathbf{x})+\varepsilon^{2} \mathbf{E}_{2}(\mathbf{x})+\cdots+\varepsilon^{k} \mathbf{E}_{k}(\mathbf{x})$ up to a residual term in $\mathcal{O}\left(\varepsilon^{k+1}\right)$. For this the equations in $(2.3)$ for $i=0, \ldots, k$ are multiplied with $\varepsilon^{i}$ and added up, and terms in $\mathcal{O}\left(\varepsilon^{k+1}\right)$ are neglected. In this way we obtain the simpler problem as

$$
\begin{array}{rlrl}
\operatorname{curl} \operatorname{curl} \mathbf{E}_{\varepsilon}^{k}-\kappa^{2} \mathbf{E}_{\varepsilon}^{k} & =i \omega \mu \mathbf{j}, & \text { in } \Omega_{-} \cup \Omega_{+}, \\
\operatorname{curl} \mathbf{E}_{\varepsilon}^{k} \times \mathbf{n}-i \kappa_{+} \mathbf{n} \times \mathbf{E}_{\varepsilon}^{k} \times \mathbf{n}=0, & \text { on } \partial \Omega,
\end{array}
$$

with the following transmission conditions on $\Gamma$ :

$$
\left(\begin{array}{c}
{\left[\mathbf{E}_{\varepsilon}^{k} \times \mathbf{n}\right]_{\Gamma}} \\
\left\{\mathbf{E}_{\varepsilon}^{k} \times \mathbf{n}\right\}_{\Gamma}
\end{array}\right)=\mathcal{G}_{k, \varepsilon}\left(\begin{array}{c}
\left\{\frac{1}{\mu}\left(\operatorname{curl} \mathbf{E}_{\varepsilon}^{k}\right)_{\mathbf{T}}\right\}_{\Gamma} \\
{\left[\left(\frac{1}{\mu} \operatorname{curl} \mathbf{E}_{\varepsilon}^{k}\right)_{\mathbf{T}}\right]_{\Gamma}}
\end{array}\right)
$$

where $\mathcal{G}_{k, \varepsilon}=\sum_{i=0}^{k} \varepsilon^{i}\left(\left[\mathcal{G}_{i}\right]_{\Gamma},\left\{\mathcal{G}_{i}\right\}_{\Gamma}\right)^{\top}$ is the truncation of the weigthed sum of operators $\mathcal{G}_{i}^{ \pm}$where the jump or mean value is taken respectively. With this derivation it 
holds at least formally $\mathbf{E}^{\varepsilon}-\mathbf{E}_{\varepsilon}^{k}=\mathcal{O}\left(\varepsilon^{k+1}\right)$. Hence, we say that $\mathbf{E}_{\varepsilon}^{k}$ is an equivalent (or approximate) model of order $k+1$.

In this paper, we give explicitly the equivalent models of order 1 and 2 in Sec. 3. Their derivations are presented in detail in Sec. 5 and Appendix A.

3. Main results. Equivalent models up to order 2. In this section we present the main results of the paper : the approximate models of order 1 (Section 3.1) and 2 (Section 3.2). Then we compare them with asymptotic models which are available in the literature (Section 3.3) and introduce a mixed variational formulation for the second order model (section 3.4).

3.1. Equivalent model of order 1 . The equivalent model of order 1 is given by the limit solution $\mathbf{E}_{0}$ of (2.1) when $\varepsilon \rightarrow 0$. The limit solution satisfies the perfect electric conductor (PEC) boundary condition on $\Gamma$ and can be defined independently in the two subdomains $\Omega_{-}, \Omega_{+}$. Hence, $\mathbf{E}_{0}^{-}=\left.\mathbf{E}_{0}\right|_{\Omega_{-}}$satisfies

$$
\begin{aligned}
\operatorname{curl} \operatorname{curl} \mathbf{E}_{0}^{-}-\kappa_{-}^{2} \mathbf{E}_{0}^{-} & =i \omega \mu_{-} \mathbf{j}_{-}, & & \text {in } \Omega_{-}, \\
\mathbf{E}_{0}^{-} \times \mathbf{n} & =0, & & \text { on } \Gamma,
\end{aligned}
$$

whereas $\mathbf{E}_{0}^{+}=\left.\mathbf{E}_{0}\right|_{\Omega_{+}}$is given by

$$
\begin{aligned}
\operatorname{curl} \operatorname{curl} \mathbf{E}_{0}^{+}-\kappa_{+}^{2} \mathbf{E}_{0}^{+} & =i \omega \mu_{+} \mathbf{j}_{+}, & & \text {in } \Omega_{+}, \\
\mathbf{E}_{0}^{+} \times \mathbf{n} & =0, & & \text { on } \Gamma, \\
\operatorname{curl} \mathbf{E}_{0}^{+} \times \mathbf{n}-i \kappa_{+} \mathbf{n} \times \mathbf{E}_{0}^{+} \times \mathbf{n} & =0, & & \text { on } \partial \Omega .
\end{aligned}
$$

The Silver-Müller boundary condition is not affected by the limiting process $\varepsilon \rightarrow 0$ and transfers simply to the limit solution $\mathbf{E}_{0}$.

3.2. Equivalent model of order 2. We define a second order approximate solution $\mathbf{E}_{\varepsilon}^{1}$, which shall be much more accurate approximation of $\mathbf{E}^{\varepsilon}$ than the limit solution $\mathbf{E}_{0}$ when $\varepsilon \rightarrow 0$. The equations defining $\mathbf{E}_{\varepsilon}^{1}$ outside the mid-surface $\Gamma$ remain the same, i.e., it solves

$$
\begin{array}{rlrl}
\operatorname{curl} \operatorname{curl} \mathbf{E}_{\varepsilon}^{1}-\kappa^{2} \mathbf{E}_{\varepsilon}^{1} & =\mathrm{i} \omega \mu \mathbf{j}, & & \text { in } \Omega_{-} \cup \Omega_{+}, \\
\operatorname{curl} \mathbf{E}_{\varepsilon}^{1} \times \mathbf{n}-\mathrm{i} \kappa_{+} \mathbf{n} \times \mathbf{E}_{\varepsilon}^{1} \times \mathbf{n}=0, & & \text { on } \partial \Omega,
\end{array}
$$

and at the mid-surface $\Gamma$ the transmission conditions

$$
\left(\begin{array}{c}
{\left[\mathbf{E}_{\varepsilon}^{1} \times \mathbf{n}\right]_{\Gamma}} \\
\left\{\mathbf{E}_{\varepsilon}^{1} \times \mathbf{n}\right\}_{\Gamma}
\end{array}\right)=\varepsilon\left(\begin{array}{cc}
\mathrm{L}_{1} & \mathrm{~L}_{3} \\
\mathrm{~L}_{3} & \mathrm{~L}_{2}
\end{array}\right)\left(\begin{array}{l}
\left\{\frac{1}{\mu}\left(\operatorname{curl} \mathbf{E}_{\varepsilon}^{1}\right)_{\mathbf{T}}\right\}_{\Gamma} \\
{\left[\left(\frac{1}{\mu} \operatorname{curl} \mathbf{E}_{\varepsilon}^{1}\right)_{\mathbf{T}}\right]_{\Gamma}}
\end{array}\right)
$$

are posed. Here, $[\cdot]_{\Gamma}$ and $\{\cdot\}_{\Gamma}$ denote the jump and averages introduced in Sec. 2.1 and $\mathrm{L}_{i}$ are differential operators given by

$$
\mathrm{L}_{i}=A_{i} \operatorname{curl}_{\Gamma} \operatorname{curl}_{\Gamma}-B_{i} \mathbb{I}, \quad i=1,2,3,
$$

in which $A_{i}, B_{i}$ are constants defined by

$$
\begin{aligned}
A_{1} & =-\omega^{-2}\left\{\left(\epsilon+\mathrm{i} \frac{\sigma}{\omega}\right)^{-1}\right\}_{\Gamma}, & B_{1} & =2 \frac{\mu_{o}}{\gamma} \tanh \left(\frac{\gamma}{2}\right)-\{\mu\}_{\Gamma}, \\
A_{2} & =\frac{A_{1}}{4}, & B_{2} & =\frac{\mu_{o}}{2 \gamma} \operatorname{coth}\left(\frac{\gamma}{2}\right)-\frac{\{\mu\}_{\Gamma}}{4}, \\
A_{3} & =-\frac{1}{4} \omega^{-2}\left[\left(\epsilon+\mathrm{i} \frac{\sigma}{\omega}\right)^{-1}\right]_{\Gamma}, & B_{3} & =-\frac{1}{4}[\mu]_{\Gamma},
\end{aligned}
$$


and

$$
\gamma=\exp \left(\frac{3 \mathrm{i} \pi}{4}\right) \sqrt{\omega \mu_{o} \tilde{\sigma}} .
$$

Equivalent model of order 2 in a "symmetric" configuration. If the electromagnetic properties on both sides of the sheet are the same, i.e., $\mu_{+}=\mu_{-}=: \mu$, $\sigma_{+}=\sigma_{-}=: \sigma$, and $\epsilon_{+}=\epsilon_{-}=: \epsilon$ (and so $\left.\kappa_{+}=\kappa_{-}=: \kappa\right)$ in $\Omega_{+} \cup \Omega_{-}$, then the transmission conditions (3.3c) of the second order model simplify to

$$
\begin{aligned}
{\left[\mathbf{E}_{\varepsilon}^{1} \times \mathbf{n}\right]_{\Gamma} } & =\varepsilon A \operatorname{curl}_{\Gamma} \operatorname{curl}_{\Gamma}\left\{\frac{1}{\mu}\left(\operatorname{curl} \mathbf{E}_{\varepsilon}^{1}\right)_{\mathbf{T}}\right\}_{\Gamma}-\varepsilon B\left\{\frac{1}{\mu}\left(\operatorname{curl} \mathbf{E}_{\varepsilon}^{1}\right)_{\mathbf{T}}\right\}_{\Gamma}, \\
\left\{\mathbf{E}_{\varepsilon}^{1} \times \mathbf{n}\right\}_{\Gamma} & =\frac{1}{4} \varepsilon A \operatorname{curl}_{\Gamma} \operatorname{curl}_{\Gamma}\left[\frac{1}{\mu}\left(\operatorname{curl} \mathbf{E}_{\varepsilon}^{1}\right)_{\mathbf{T}}\right]_{\Gamma}-\varepsilon C\left[\frac{1}{\mu}\left(\operatorname{curl} \mathbf{E}_{\varepsilon}^{1}\right)_{\mathbf{T}}\right]_{\Gamma},
\end{aligned}
$$

where

$$
A=-\omega^{-2}\left(\epsilon+\mathrm{i} \frac{\sigma}{\omega}\right)^{-1}, \quad B=\frac{2 \mu_{o}}{\gamma} \tanh \left(\frac{\gamma}{2}\right)-\mu, \quad C=\frac{\mu_{o}}{2 \gamma} \operatorname{coth}\left(\frac{\gamma}{2}\right)-\frac{\mu}{4} .
$$

Equivalent model of order 2 set on the two surfaces $\Gamma_{+}^{\varepsilon}$ and $\Gamma_{-}^{\varepsilon}$. The transmission conditions (3.3c) of order 2 are set on the mid-surface $\Gamma$. Alternatively, an equivalent model can be defined in the exterior of the sheet and with transmission conditions across the two distinct surfaces $\Gamma_{+}^{\varepsilon}$ and $\Gamma_{-}^{\varepsilon}$ of the conducting layer. Details of this definition by asymptotic expansion can be found for other thin layer models in electromagnetics in [9]. For the model of the thin and highly conducting layer the transmission conditions of order 2 across the two surfaces of the layer are given by

$$
\begin{aligned}
{\left[\widetilde{\mathbf{E}}_{\varepsilon}^{1} \times \mathbf{n}\right]_{\Gamma^{\varepsilon}} } & =-\varepsilon \widetilde{B}\left\{\frac{1}{\mu}\left(\operatorname{curl} \widetilde{\mathbf{E}}_{\varepsilon}^{1}\right)_{\mathbf{T}}\right\}_{\Gamma^{\varepsilon}}, \\
\left\{\widetilde{\mathbf{E}}_{\varepsilon}^{1} \times \mathbf{n}\right\}_{\Gamma^{\varepsilon}} & =-\varepsilon \widetilde{C}\left[\frac{1}{\mu}\left(\operatorname{curl} \widetilde{\mathbf{E}}_{\varepsilon}^{1}\right)_{\mathbf{T}}\right]_{\Gamma^{\varepsilon}},
\end{aligned}
$$

whose parameters are given by

$$
\widetilde{B}=\frac{2 \mu_{o}}{\gamma} \tanh \left(\frac{\gamma}{2}\right), \quad \widetilde{C}=\frac{\mu_{o}}{2 \gamma} \operatorname{coth}\left(\frac{\gamma}{2}\right),
$$

and $[\mathbf{v}]_{\Gamma^{\varepsilon}}$ and $\{\mathbf{v}\}_{\Gamma^{\varepsilon}}$ are the jump and mean-value of $\mathbf{v}$ across the thin layer

$$
[\mathbf{v}]_{\Gamma^{\varepsilon}}=\left.\mathbf{v}\right|_{\Gamma_{+}^{\varepsilon}}-\left.\mathbf{v}\right|_{\Gamma_{-}^{\varepsilon}}, \quad\{\mathbf{v}\}_{\Gamma^{\varepsilon}}=\left.\frac{1}{2} \mathbf{v}\right|_{\Gamma_{+}^{\varepsilon}}+\left.\frac{1}{2} \mathbf{v}\right|_{\Gamma_{-}^{\varepsilon}},
$$

They take a much simpler form than the transmission conditions (3.3c) on the midsurface, especially, due to the absence of the second order surface curl operators. Moreover, the parameters $\widetilde{B}$ and $\widetilde{C}$ of these transmission conditions do not depend on the electromagnetic properties outside the thin layer. Even so the equivalent model with transmission conditions (3.8) across the two surfaces of the conductor shall lead to the same order in $\varepsilon$ as the equivalent model with the transmission conditions (3.3c). For standard finite element methods the meshes of the two subdomains $\Omega_{ \pm}^{\varepsilon}$ have to be compatible in the way that the faces of the cells on $\Gamma_{-}^{\varepsilon}$ have to match with those on $\Gamma_{+}^{\varepsilon}$. This restriction might be lowered by using appropriate mortar finite element methods [35]. 


\subsection{Comparison with equivalent models in the literature.}

3.3.1. The Levi-Civita conditions. Already in 1902 Levi-Civita has proposed conditions [17] replacing thin conducting layers. He assumed that the tangential components of the electric field $\mathbf{E}_{L C}$ are continuous, whereas the tangential components of the magnetic field $\mathbf{H}_{L C}=\frac{1}{\mu} \operatorname{curl} \mathbf{E}_{L C}$ are discontinuous due to induced currents $\varepsilon^{-2} \tilde{\sigma} \mathbf{E}_{L C}$. Adopted to the more general setting of this article the conditions are given by

$$
\begin{aligned}
{\left[\mathbf{E}_{L C} \times \mathbf{n}\right]_{\Gamma} } & =0, \\
\left\{\mathbf{E}_{L C} \times \mathbf{n}\right\}_{\Gamma} & =-\varepsilon C_{L C}\left[\frac{1}{\mu}\left(\operatorname{curl} \mathbf{E}_{L C}\right)_{\mathbf{T}}\right]_{\Gamma},
\end{aligned}
$$

where $C_{L C}=\mu_{o} / \gamma^{2}$. It can be proven that they arise as the limit conditions for $\varepsilon \rightarrow 0$ if the conductivity $\sigma_{0}^{\varepsilon}$ in the thin layer is scaled like $\varepsilon^{-1}$ instead of $\varepsilon^{-2}$. This has been observed in [32] for thin conducting layers in two dimensions. In this limit process the terms $\varepsilon A_{i}, i=1,2,3$ and $\varepsilon B_{i}, i=1,3$ for the general setting become zero and, as $\gamma \rightarrow 0$, the parameter $B_{2}$ tends to $C_{L C}$. This can be also seen for the symmetric configuration, where $\varepsilon A$ and $\varepsilon B$ become zero in the limit and $C$ tends to $C_{L C}$.

3.3.2. The thin layer boundary conditions [34]. In the computational electromagnetics community the thin layer boundary conditions by Mayergoyz and coworkers $[34,18,13]$ are the most prominent transmission conditions. They are believed to be superior to other conditions like those by Levi-Civita since they incorporate the boundary layer behaviour due to the skin effect inside the thin layer. In comparison to the Levi-Civita conditions also the (tangential component of the) electric field is discontinuous across $\Gamma$. For the setting of the article the thin layer boundary conditions are given by

$$
\begin{array}{r}
{\left[\widetilde{\mathbf{E}}_{\varepsilon}^{1} \times \mathbf{n}\right]_{\Gamma}=-\varepsilon \widetilde{B}\left\{\frac{1}{\mu}\left(\operatorname{curl} \widetilde{\mathbf{E}}_{\varepsilon}^{1}\right)_{\mathbf{T}}\right\}_{\Gamma},} \\
\left\{\widetilde{\mathbf{E}}_{\varepsilon}^{1} \times \mathbf{n}\right\}_{\Gamma}=-\varepsilon \widetilde{C}\left[\frac{1}{\mu}\left(\operatorname{curl} \widetilde{\mathbf{E}}_{\varepsilon}^{1}\right)_{\mathbf{T}}\right]_{\Gamma},
\end{array}
$$

where $\widetilde{B}$ and $\widetilde{C}$ has been defined in (3.9). They differ from the transmission conditions (3.11) only in the fact that the jump $[\cdot]_{\Gamma^{\varepsilon}}$ and mean $\{\cdot\}_{\Gamma^{\varepsilon}}$ across the thin layer are replaced by the jump $[\cdot]_{\Gamma}$ and $\{\cdot\}_{\Gamma}$ across the mid-surface. Indeed the transmission conditions (3.8) appear in an intermediate step when deriving the thin layer boundary conditions (cf. e.g. [13, Eqs. (11)-(10')]), but then the two surfaces are identified to each other. This identification is practical for boundary integral formulations as in $[13,30]$, which do not have to deal with two close surfaces. However, this identification harms the modelling error, a fact which has been reported in two dimensions in [28].

3.4. Mixed variational formulation. The transmission condition (3.3c) is in its general form of Wentzel type when regarded as a Poincaré-Steklov map $\mathbf{H}_{\mathbf{T}}{ }^{-}$to$\mathbf{E} \times \mathbf{n}$ and tends to the PEC boundary condition for $\varepsilon \rightarrow 0$. Therefore, we use the mixed variational formulation with additional unknowns $\boldsymbol{\lambda}_{\varepsilon}$ and $\boldsymbol{\mu}_{\varepsilon}$ defined as

$$
\boldsymbol{\lambda}_{\varepsilon}=\left\{\frac{1}{\mu}\left(\operatorname{curl} \mathbf{E}_{\varepsilon}^{1}\right)_{\mathbf{T}}\right\}_{\Gamma} \quad \text { and } \quad \boldsymbol{\mu}_{\varepsilon}=\left[\frac{1}{\mu}\left(\operatorname{curl} \mathbf{E}_{\varepsilon}^{1}\right)_{\mathbf{T}}\right]_{\Gamma},
$$


in which the Poincaré-Steklov map (3.3c) is incorporated in weak sense. This formulation is also known as saddle point problem with penalty term $[3, \S 4$, pp. $138 \mathrm{ff}]$. In this formulation we search for $\mathbf{E}_{\varepsilon}^{1}$ in the Hilbert space

$$
\mathbf{V}=\left\{\mathbf{E} \in \mathbf{H}\left(\operatorname{curl}, \Omega_{ \pm}\right), \mathbf{E} \times \mathbf{n} \in \mathbf{L}_{t}^{2}(\partial \Omega)\right\},
$$

and for $\boldsymbol{\lambda}_{\varepsilon}$ and $\boldsymbol{\mu}_{\varepsilon}$ in the Hilbert space

$$
\mathbf{W}=\mathrm{TH}\left(\operatorname{curl}_{\Gamma}, \Gamma\right) .
$$

Hence, we obtain the mixed variational formulation : Find $\left(\mathbf{E}_{\varepsilon}^{1}, \boldsymbol{\lambda}_{\varepsilon}, \boldsymbol{\mu}_{\varepsilon}\right) \in \mathbf{V} \times \mathbf{W} \times \mathbf{W}$ such that for all $\left(\mathbf{U}, \boldsymbol{\xi}_{1}, \boldsymbol{\xi}_{2}\right) \in \mathbf{V} \times \mathbf{W} \times \mathbf{W}$

$$
\begin{aligned}
& \int_{\Omega_{+} \cup \Omega_{-}} \frac{1}{\mu} \operatorname{curl} \mathbf{E}_{\varepsilon}^{1} \cdot \operatorname{curl} \overline{\mathbf{U}}-\frac{\kappa^{2}}{\mu} \mathbf{E}_{\varepsilon}^{1} \cdot \overline{\mathbf{U}} \mathrm{d} \mathbf{x}-\mathrm{i} \frac{\kappa_{+}}{\mu_{+}} \int_{\partial \Omega} \mathbf{E}_{\varepsilon}^{1} \times \mathbf{n} \cdot \overline{\mathbf{U}} \times \mathbf{n} \mathrm{d} S \\
& -\int_{\Gamma}\left(\begin{array}{l}
\mathbf{n} \times \boldsymbol{\lambda}_{\varepsilon} \\
\mathbf{n} \times \boldsymbol{\mu}_{\varepsilon}
\end{array}\right) \cdot\left(\begin{array}{l}
{\left[\overline{\mathbf{U}}_{\mathbf{T}}\right]} \\
\left\{\overline{\mathbf{U}}_{\mathbf{T}}\right\}
\end{array}\right) \mathrm{d} S=\mathrm{i} \omega \int_{\Omega} \mathbf{j} \cdot \overline{\mathbf{U}} \mathrm{d} \mathbf{x},
\end{aligned}
$$

and

$$
\int_{\Gamma}\left(\begin{array}{c}
{\left[\mathbf{n} \times \mathbf{E}_{\varepsilon}^{1}\right]} \\
\left.\mathbf{n} \times \mathbf{E}_{\varepsilon}^{1}\right\}
\end{array}\right) \cdot\left(\begin{array}{l}
\overline{\boldsymbol{\xi}}_{1} \\
\overline{\boldsymbol{\xi}}_{2}
\end{array}\right)+\varepsilon \mathrm{A}\left(\begin{array}{c}
\operatorname{curl}_{\Gamma} \boldsymbol{\lambda}_{\varepsilon} \\
\operatorname{curl}_{\Gamma} \boldsymbol{\mu}_{\varepsilon}
\end{array}\right) \cdot\left(\begin{array}{c}
\operatorname{curl}_{\Gamma} \overline{\boldsymbol{\xi}}_{1} \\
\operatorname{curl}_{\Gamma} \overline{\boldsymbol{\xi}}_{2}
\end{array}\right)-\varepsilon \mathrm{B}\left(\begin{array}{c}
\boldsymbol{\lambda}_{\varepsilon} \\
\boldsymbol{\mu}_{\varepsilon}
\end{array}\right) \cdot\left(\begin{array}{l}
\overline{\boldsymbol{\xi}}_{1} \\
\overline{\boldsymbol{\xi}}_{2}
\end{array}\right) \mathrm{d} S=0 .
$$

Here, $A$ and $B$ are matrices given by

$$
\mathrm{A}=\left(\begin{array}{cc}
A_{1} & A_{3} \\
A_{3} & A_{2}
\end{array}\right) \quad \text { and } \quad \mathrm{B}=\left(\begin{array}{cc}
B_{1} & B_{3} \\
B_{3} & B_{2}
\end{array}\right)
$$

where the constants $A_{i}, B_{i}$ are defined in (3.4).

REMARK 3.1. In general, the first term in (3.13b) is nothing but the sum of two duality products $\int_{\Gamma}\left[\mathbf{n} \times \mathbf{E}_{\varepsilon}^{1}\right] \cdot \overline{\boldsymbol{\xi}}_{1} \mathrm{~d} S$ and $\int_{\Gamma}\left\{\mathbf{n} \times \mathbf{E}_{\varepsilon}^{1}\right\} \cdot \overline{\boldsymbol{\xi}}_{2} \mathrm{~d} S$ between two function spaces $\mathrm{TH}^{-\frac{1}{2}}\left(\operatorname{div}_{\Gamma}, \Gamma\right)$ and $\mathrm{TH}\left(\operatorname{curl}_{\Gamma}, \Gamma\right)$, which coincide with the $\mathrm{L}^{2}(\Gamma)$ scalar product for smooth functions.

Equivalent model of order 2 in a "symmetric" configuration. In this case the transmission conditions (3.3c) split up. They are given by (3.6)

$$
\begin{aligned}
{\left[\mathbf{E}_{\varepsilon}^{1} \times \mathbf{n}\right]_{\Gamma} } & =\varepsilon A \operatorname{curl}_{\Gamma} \operatorname{curl}_{\Gamma} \boldsymbol{\lambda}_{\varepsilon}-\varepsilon B \boldsymbol{\lambda}_{\varepsilon} \\
\left\{\mathbf{E}_{\varepsilon}^{1} \times \mathbf{n}\right\}_{\Gamma} & =\varepsilon \frac{A}{4} \operatorname{curl}_{\Gamma} \operatorname{curl}_{\Gamma} \boldsymbol{\mu}_{\varepsilon}-\varepsilon C \boldsymbol{\mu}_{\varepsilon},
\end{aligned}
$$

where constants $A, B, C$ are defined in (3.7). The variational formulation is as (3.13) where $(3.13 \mathrm{~b})$ is replaced by

$$
\begin{aligned}
& \int_{\Gamma}\left[\mathbf{n} \times \mathbf{E}_{\varepsilon}^{1}\right] \cdot \overline{\boldsymbol{\xi}}_{1} \mathrm{~d} S+\varepsilon A \int_{\Gamma} \operatorname{curl}_{\Gamma} \boldsymbol{\lambda}_{\varepsilon} \operatorname{curl}_{\Gamma} \overline{\boldsymbol{\xi}}_{1} \mathrm{~d} S-\varepsilon B \int_{\Gamma} \boldsymbol{\lambda}_{\varepsilon} \cdot \overline{\boldsymbol{\xi}}_{1} \mathrm{~d} S=0 \\
& \int_{\Gamma}\left\{\mathbf{n} \times \mathbf{E}_{\varepsilon}^{1}\right\} \cdot \overline{\boldsymbol{\xi}}_{2} \mathrm{~d} S+\varepsilon \frac{A}{4} \int_{\Gamma} \operatorname{curl}_{\Gamma} \boldsymbol{\mu}_{\varepsilon} \operatorname{curl}_{\Gamma} \overline{\boldsymbol{\xi}}_{2} \mathrm{~d} S-\varepsilon C \int_{\Gamma} \boldsymbol{\mu}_{\varepsilon} \cdot \overline{\boldsymbol{\xi}}_{2} \mathrm{~d} S=0
\end{aligned}
$$


4. Numerical investigation of the equivalent model properties. This section illustrates the different equivalent models for both the diffraction and shielding problem of a incoming plane wave by a spherical thin conducting layer (Sec. 4.1) and the shielding problem of a Gaussian current source by a flat layer (Sec. 4.2). Numerical convergence rates show the order of accuracy for each equivalent model. Section 4.3 presents numerical results for the robustness of each equivalent model with respect to the parameter $\tilde{\sigma}$, which corresponds to the skin-depth-to-thickness ratio of the sheet $d_{\text {skin }} / \varepsilon$ for fixed frequency since $d_{\text {skin }}=\varepsilon \sqrt{2 /\left(\omega \mu_{o} \widetilde{\sigma}\right)}$.
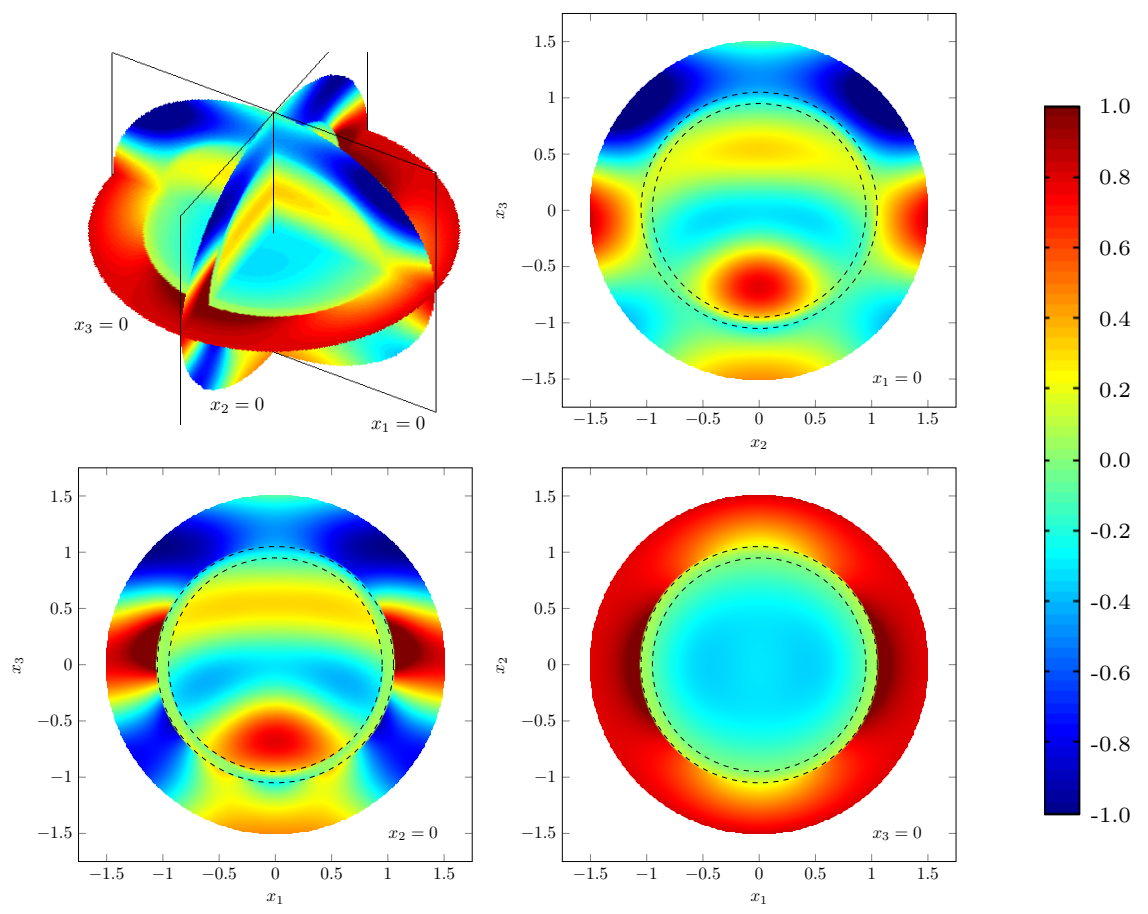

FIG. 4.1. Scattering by a spherical thin layer of radius 1 and thickness $\varepsilon=0.1$ inside a spherical domain of radius 1.5. The real part of the $\mathbf{e}_{1}$ component of the total field is shown in different slices.

4.1. Configuration of a spherical layer. The model is tested for a spherical layer whose mid-surface has radius 1, the computational domain is the ball of radius 1.5. The following parameters are chosen

$$
\begin{aligned}
& \epsilon_{+}=1 \text {, } \\
& \mu_{+}=1 \text {, } \\
& \sigma_{+}=0, \\
& \epsilon_{-}=2 \text {, } \\
& \mu_{-}=1.5 \text {, } \\
& \sigma_{-}=0.5 \text {, } \\
& \epsilon_{o}=3.5 \text {, } \\
& \mu_{o}=2.0 \text {, } \\
& \tilde{\sigma}=0.5 \text {. }
\end{aligned}
$$

They are chosen such that all the coefficients $A_{i}$ and $B_{i}$ are different from 0 . The source is here an incident plane wave, $\mathbf{E}^{\mathrm{inc}}=\exp \left(-\mathrm{i} \omega x_{3}\right) \mathbf{e}_{1}$, i.e., there is no current source, and Silver-Müller radiation condition applies to the scattered field $\mathbf{E}_{\varepsilon}^{k, \mathrm{sc}}=$ $\mathbf{E}_{\varepsilon}^{k}-\mathbf{E}^{\text {inc }}$. The exact solution, which can be computed analytically with spherical Bessel functions (cf. e.g. [23, Sec. 2.4]), is shown for $\omega=\pi$ in Fig. 4.1.

Nédélec's elements of the first kind on hexahedral curved elements are used to compute the approximate solution (see [6] for more details). They satisfy tangential 


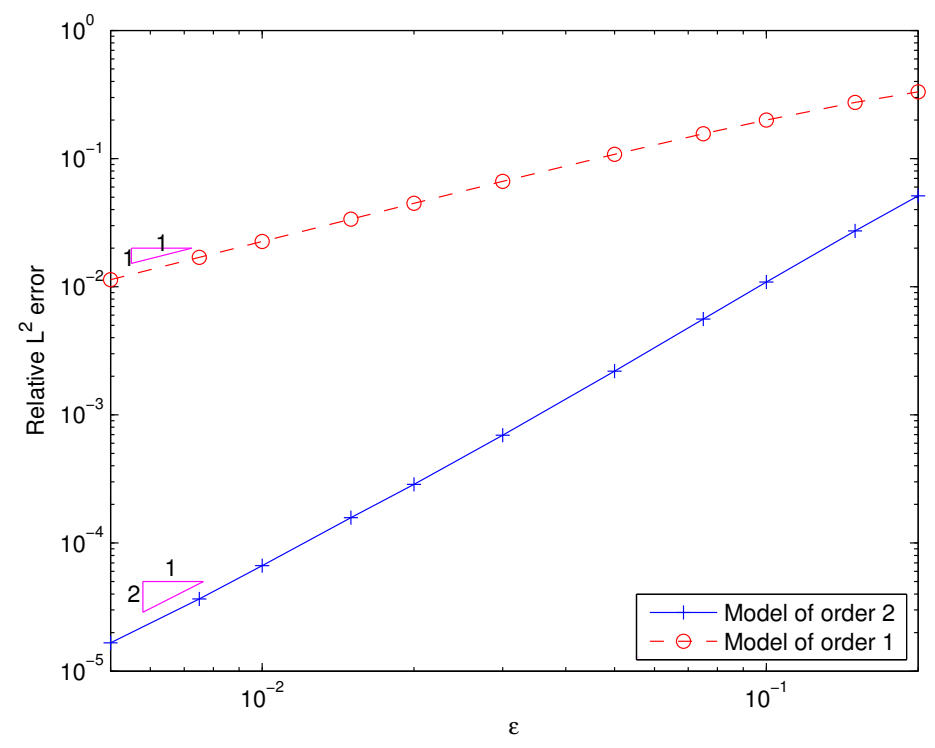

FIG. 4.2. Relative $\mathbf{L}^{2}$ errors of the solutions $\mathbf{E}_{0}, \mathbf{E}_{\varepsilon}^{1}$ of the equivalent models of order 1 and 2 versus the thickness $\varepsilon$ (lower frequency $\omega=\frac{\pi}{5}$ ).

continuity over all element boundaries except the faces on the mid-surface of the sheet. We have used sixth order elements on a fine enough mesh such that the discretisation error is negligible over the modelling errors. On the mid-surface of the sheet the equivalent conditions of order 1 (which are the PEC boundary conditions (3.1b) and $(3.2 \mathrm{~b})$ ) or order 2 (see (3.3c)) are applied, where we use edge elements for the auxiliary variables $\boldsymbol{\lambda}_{\varepsilon}$ and $\boldsymbol{\mu}_{\varepsilon}$ on the interface $\Gamma$ (the finite element space for auxiliary variables is the tangential trace of the 3-D finite element space used for the electrical field).

We have performed the numerical experiments for two frequencies, a lower frequency $\omega=\frac{\pi}{5}$ and a higher frequency $\omega=\pi$. Note, that the skin depth $d_{\text {skin }}=$ $\varepsilon \sqrt{2 /\left(\omega \mu_{o} \widetilde{\sigma}\right)}$ inside the thin conducting sheet, which scales in this asymptotic regime linearly with $\varepsilon$, is $1.7841 \varepsilon$ for $\omega=\frac{\pi}{5}$ and $d_{\text {skin }}=0.7979 \varepsilon$ for $\omega=\pi$. The relative $\mathbf{L}^{2}$ errors (computed in $\Omega_{\text {ext }}$, the domain $\Omega$ excluding the spherical crown $0.9<r<1.1$ )

$$
\frac{\left\|\mathbf{E}_{0}-\mathbf{E}^{\varepsilon}\right\|_{L^{2}\left(\Omega_{\mathrm{ext}}\right)}}{\left\|\mathbf{E}^{\varepsilon}\right\|_{L^{2}\left(\Omega_{\mathrm{ext}}\right)}}, \quad \frac{\left\|\mathbf{E}_{\varepsilon}^{1}-\mathbf{E}^{\varepsilon}\right\|_{L^{2}\left(\Omega_{\mathrm{ext}}\right)}}{\left\|\mathbf{E}^{\varepsilon}\right\|_{L^{2}\left(\Omega_{\mathrm{ext}}\right)}}
$$

of the equivalent models of order 1 and order 2 are shown in Fig. 4.2 for the lower frequency and in Fig. 4.3 for higher frequency as a function of the thickness parameter $\varepsilon$. The model of order 1 with PEC boundary condition, see (3.1)-(3.2), shows in the numerical experiments only a convergence as $O(\varepsilon)$ for both frequencies (see dashed line in Fig. 4.2 and Fig. 4.3) as expected by the asymptotic expansion. When the model of order 2, see (3.3), is used for the lower frequency we observe the expected second-order convergence (see solid line in Fig. 4.2). For the larger frequency we observe for the investigated thicknesses $\varepsilon$ a local convergence approximately as $O\left(\varepsilon^{2.5}\right)$ (see solid line in Fig. 4.3). However, we expect that this is a pre-asymptotic behaviour and such an additional convergence rate cannot be expected in general, and a second-order convergence will be recovered for smaller values of $\varepsilon$. These ex- 


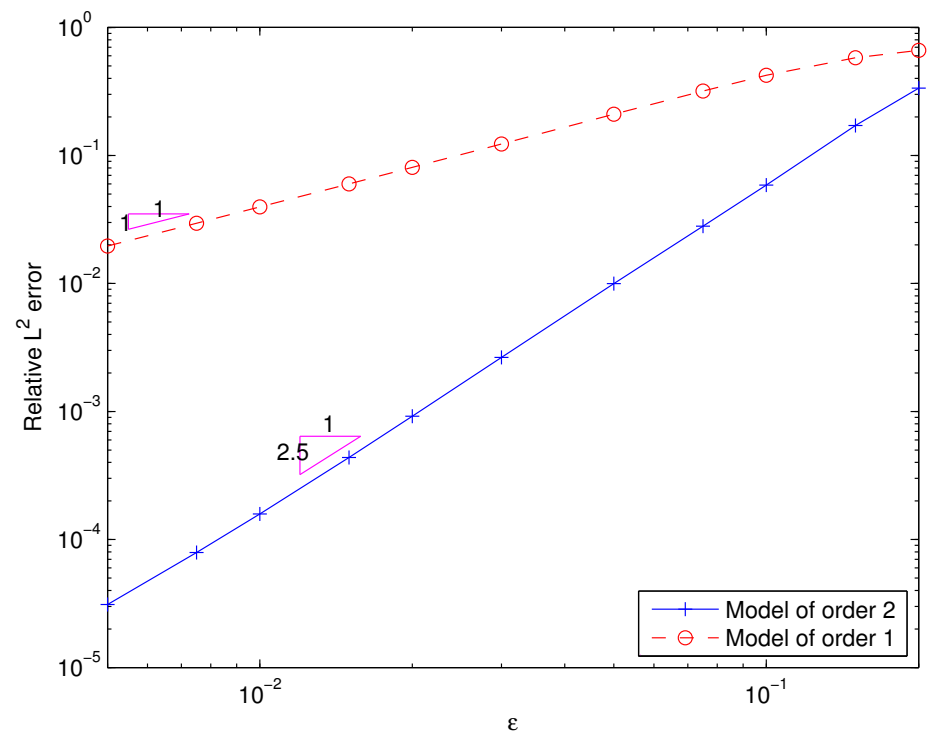

FIG. 4.3. Relative $\mathbf{L}^{2}$ errors of the solutions $\mathbf{E}_{0}, \mathbf{E}_{\varepsilon}^{1}$ of the equivalent models of order 1 and 2 versus the thickness $\varepsilon$ (higher frequency $\omega=\pi$ ).

periments show that the equivalent models do not provide higher convergence orders than the expected ones. Hence, only an equivalent model of even higher order, i.e., at least order 3, which then involves curvature terms, may in general exhibit higher convergence.

Nevertheless, we will show in the next section (see Fig. 4.4) that third-order convergence is observed for a special case, namely that of a flat layer.

4.2. Configuration of a flat layer. In this simulation, the computational domain is the cube $[-5,5]^{3}$, and the conducting sheet is centered at $z=0$. The material parameters are the same as for the spherical layer, see (4.1), where $\epsilon_{+}, \mu_{+}, \sigma_{+}$apply for $z>0$ and $\epsilon_{-}, \mu_{-}, \sigma_{-}$for $z<0$. The pulsation is given as $\omega=\frac{2 \pi}{5}$. Here, we use a current source $\mathbf{j}$ which is a Gaussian centered at $(0,0,2.5)$ and truncated at a distance of 1.5 from the center, and which is, hence, null in the thin layer. Periodic boundary conditions are applied on lateral surfaces and the Silver-Müller condition is applied on surfaces $z= \pm 5$. In Figure 4.4 we can see that the equivalent model $\mathbf{E}_{\varepsilon}^{1}$ converges towards the exact solution even as $O\left(\varepsilon^{3}\right)$ as observed for flat sheets in 2-D (see [28]).

4.3. Robustness of the equivalent models versus the parameter $\tilde{\sigma}$. Finally, the robustness of each equivalent model [28] with respect to $\tilde{\sigma}$ is studied. For illustration we show in Figure 4.5 the relative $\mathbf{L}^{2}$ error in dependence of the parameter $\tilde{\sigma}$ for $\omega=\pi$ and the above introduced thin spherical layer, for which we set $\varepsilon=0.01$ or $\varepsilon=0.02$, respectively. Since for fixed frequency, sheet thickness and other material parameters the skin depth is a function of $\tilde{\sigma}$, varying $\tilde{\sigma}$ corresponds to a variation of the skin depth, and so these experiments show the accuracy for a large range of skin depths, from very small to very large.

We observe that the model of order 1 is not robust in $\tilde{\sigma}$ since the error does not decrease with $\varepsilon$ for $\tilde{\sigma} \rightarrow 0$, i.e., for large skin depths. We observe an error reduction 


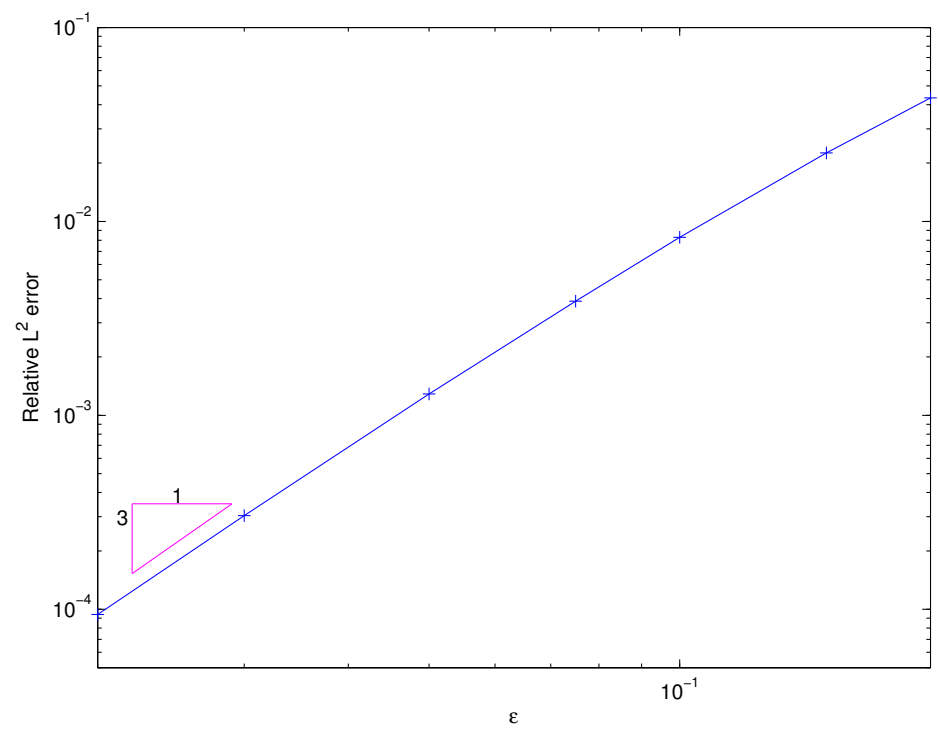

FIG. 4.4. Relative $\mathbf{L}^{2}$ error of the solutions $\mathbf{E}_{\varepsilon}^{1}$ of the model of order 2, see (3.3) in Sec. 3.2 versus the thickness $\varepsilon$ for the flat sheet.

in $\varepsilon$ (namely by a factor 2 for $\tilde{\sigma} \rightarrow \infty$, i.e., the error behaves like $\varepsilon$ ) only if $\tilde{\sigma}$ is large enough. In difference the model of order 2 is robust in $\tilde{\sigma}$. We observe an error reduction for any small or large value of $\tilde{\sigma}$, or equivalently for large and small skin depths. The error reduction is higher for large values of $\tilde{\sigma}$ (factor 4 for $\tilde{\sigma} \rightarrow \infty$, i.e., the error behaves like $\varepsilon^{2}$ ) than for small ones (factor 2 for $\tilde{\sigma} \rightarrow 0$, i.e., the error behaves like $\varepsilon$ ). In any case, the equivalent model of order 2 shows a higher accuracy than that of order 1 .

5. A multiscale expansion for the electric field. In the guidelines on the derivation of the equivalent transmission conditions in Sect. 2.4 we have already argued that this derivation is based on an asymptotic expansion for the electric field $\mathbf{E}^{\varepsilon}(2.1)$ inside and outside the sheet. More precisely, we search $\mathbf{E}^{\varepsilon}$ as the asymptotic expansion (2.2), which is

$$
\begin{array}{ll}
\mathbf{E}^{\varepsilon}(\mathbf{x}) \approx \mathbf{E}_{0}(\mathbf{x})+\varepsilon \mathbf{E}_{1}(\mathbf{x})+\varepsilon^{2} \mathbf{E}_{2}(\mathbf{x})+\cdots & \text { for a.e. } \quad \mathbf{x} \in \Omega_{-}^{\varepsilon} \cup \Omega_{+}^{\varepsilon}, \\
\mathbf{E}^{\varepsilon}(\mathbf{x}) \approx \mathfrak{E}_{0}\left(y_{\alpha}, \frac{h}{\varepsilon}\right)+\varepsilon \mathfrak{E}_{1}\left(y_{\alpha}, \frac{h}{\varepsilon}\right)+\cdots & \text { for a.e. } \quad \mathbf{x} \in \Omega_{o}^{\varepsilon} .
\end{array}
$$

In this section, we will derive the terms of this asymptotic expansion step by step up to order 2 as well as their governing equations, having in mind that the electric 


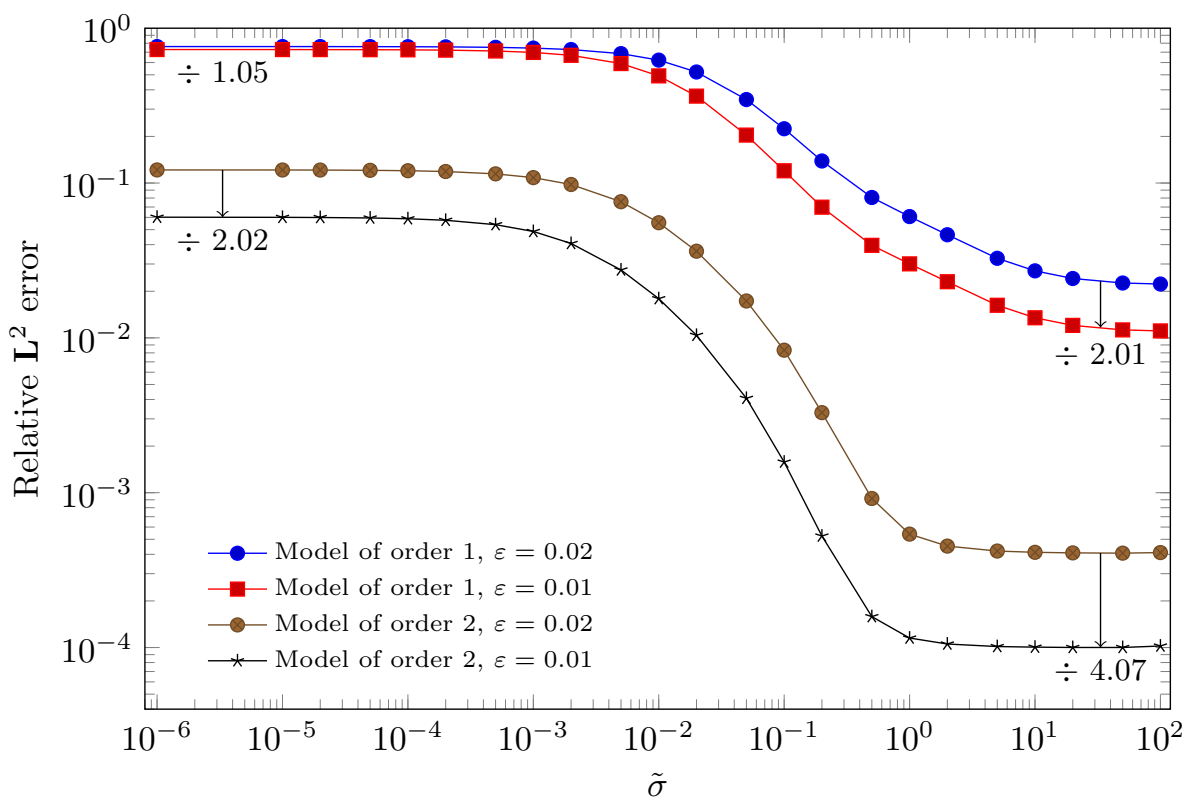

FIG. 4.5. Relative $\mathbf{L}^{2}$ error of the solutions $\mathbf{E}_{0}$ and $\mathbf{E}_{\varepsilon}^{1}$ of the equivalent models of order 1 and order 2 versus the parameter $\tilde{\sigma}$ (in abscissa) for $\varepsilon=0.01$ and $\varepsilon=0.02$.

field $\mathbf{E}^{\varepsilon}$ satisfies the following Maxwell transmission problem

$$
\begin{array}{rlrl}
\operatorname{curlcurl} \mathbf{E}_{ \pm}^{\varepsilon}-\kappa_{ \pm}^{2} \mathbf{E}_{ \pm}^{\varepsilon} & =i \omega \mu_{ \pm} \mathbf{j}_{ \pm} & \text {in } \Omega_{ \pm}^{\varepsilon} \\
\operatorname{curlcurl} \mathbf{E}_{o}^{\varepsilon}-\left(\kappa_{o}^{\varepsilon}\right)^{2} \mathbf{E}_{o}^{\varepsilon}=0 & \text { in } \Omega_{o}^{\varepsilon}, \\
\frac{1}{\mu_{ \pm}} \operatorname{curl} \mathbf{E}_{ \pm}^{\varepsilon} \times \mathbf{n}=\frac{1}{\mu_{o}} \operatorname{curl} \mathbf{E}_{o}^{\varepsilon} \times \mathbf{n} & \text { on } \Gamma_{ \pm}^{\varepsilon}, \\
\mathbf{E}_{ \pm}^{\varepsilon} \times \mathbf{n}=\mathbf{E}_{o}^{\varepsilon} \times \mathbf{n} & \text { on } \Gamma_{ \pm}^{\varepsilon}, \\
\operatorname{curl} \mathbf{E}_{+}^{\varepsilon} \times \mathbf{n}-i \kappa_{+} \mathbf{n} \times \mathbf{E}_{+}^{\varepsilon} \times \mathbf{n}=0 & \text { on } \partial \Omega
\end{array}
$$

This derivation is order by order and for each order $n$ it is in four steps:

(i) Writing the operator curl curl in the domain $\Omega_{o}^{\varepsilon}$ in local coordinates $\left(y_{\alpha}, h\right)[4]$ and performing the change of variable $Y_{3}=\varepsilon^{-1} h$, i.e., rewriting it in in $\left(y_{\alpha}, Y_{3}\right)$-coordinates, leads to an algebraic equation fixing the normal component $\mathfrak{e}_{n}$ of $\mathfrak{E}_{n}$ and a differential equation for the two tangential components $\mathfrak{E}_{n}$, which are not completely defined yet.

(ii) We expand the far field term $\mathbf{E}_{n}$ at the two surfaces $\Gamma_{ \pm}^{\varepsilon}$ of the conductor around its mid-surface $\Gamma$ using the Taylor expansion.

(iii) Using the Neumann continuity condition on $\Gamma_{ \pm}^{\varepsilon}$ together with the Taylor expansion of $\mathbf{E}_{n}$ we obtain Neumann boundary conditions for the tangential components $\mathfrak{E}_{n}$ inside the sheet. They can now be explicitly defined as a function of the terms $\mathbf{E}_{k}, k=0, \ldots, n-1$ of lower orders only.

(iv) Using the Dirichlet continuity condition on $\Gamma_{ \pm}^{\varepsilon}$ and the Taylor expansion of $\mathbf{E}_{n}$ we obtain Dirichlet conditions for $\mathbf{E}_{n}$ on $\Gamma$, which depend on $\mathbf{E}_{k}, k=$ $0, \ldots, n-1$ and their derivatives on $\Gamma$. 
We expand the "electric" Maxwell operators inside the thin layer $\Omega_{o}^{\varepsilon}$ in powers of $\varepsilon$, in Section 5.1. We deduce in Section 5.2 the equations satisfied by the electric profiles $\mathfrak{E}_{n}$ and the far field terms $\mathbf{E}_{n}^{ \pm}$. We derive explicitly the first terms in Section 5.3.

5.1. Expansion of differential operators inside the conductor. Due to the small thickness of the conductor the derivatives in normal and the tangential directions scale differently in $\varepsilon$. Hence, it is convenient to use a local normal coordinate system in $\Omega_{o}^{\varepsilon}$, see e.g., [4, App. A.1]. For this coordinate system we call $D_{\alpha}$ the covariant derivative on the mean surface $\Gamma$ and $\partial_{3}^{h}$ is the partial derivative with respect to the normal coordinate $y_{3}=h$. Let furthermore $a_{\alpha \beta}(h)$ be the metric tensor of the manifold $\Gamma_{h}$, which is the surface contained in $\Omega_{2}^{\varepsilon}$ at a distance $h$ of $\Gamma$. The metric tensor in such a coordinate system writes [4, App. A.1, Eq. (A.7)]

$$
a_{\alpha \beta}(h)=a_{\alpha \beta}-2 b_{\alpha \beta} h+b_{\alpha}^{\gamma} b_{\gamma \beta} h^{2},
$$

and its inverse expands in power series of $h$

$$
a^{\alpha \beta}(h)=a^{\alpha \beta}+2 b^{\alpha \beta} h+\mathcal{O}\left(h^{2}\right) .
$$

Subsequently, we use a property of the covariant derivative, that it acts on scalar functions $\mathfrak{e}$ like the partial derivative: $D_{\alpha} \mathfrak{e}=\partial_{\alpha} \mathfrak{e}$.

We denote by $\mathbf{L}\left(y_{\alpha}, h ; D_{\alpha}, \partial_{3}^{h}\right)$ the second order Maxwell operator

$$
\operatorname{curl} \operatorname{curl}-\left(\kappa_{o}^{\varepsilon}\right)^{2} \mathbb{I}
$$

in $\Omega_{o}^{\varepsilon}$ in the normal coordinate system and by $\mathbf{B}\left(y_{\alpha}, h ; D_{\alpha}, \partial_{3}^{h}\right)=\left(\mathbf{B}_{\alpha}\left(y_{\alpha}, h ; D_{\alpha}, \partial_{3}^{h}\right), 0\right)$ the tangent trace operator curl $\times \times \mathbf{n}$ on $\Gamma_{ \pm}^{\varepsilon}$, with

$$
\mathbf{B}_{\alpha}\left(y_{\alpha}, h ; D_{\alpha}, \partial_{3}^{h}\right) \mathfrak{E}=\partial_{3}^{h} \mathfrak{E}_{\alpha}-D_{\alpha} \mathfrak{e},
$$

for $\mathfrak{E}=\left(\mathfrak{E}_{\alpha}, \mathfrak{e}\right)$, see $[4$, App. A, $\S$ A.4]. The operators $\mathbf{L}$ and $\mathbf{B}$ expand in power series of $h$ with intrinsic coefficients with respect to $\Gamma$, see [4].

Now, we scale the normal coordinate $Y_{3}=\varepsilon^{-1} h$ to obtain a coordinate, this is $Y_{3}$, which does not change with $\varepsilon$. We use from now on the same symbol $\mathfrak{E}$ for three-dimensional one-form field in these scaled coordinates and call $\mathbf{L}[\varepsilon]$ and $\mathbf{B}[\varepsilon]$ the respective three-dimensional harmonic Maxwell operators in $\Omega_{o}^{\varepsilon}$. These operators expand in powers of $\varepsilon$

$$
\mathbf{L}[\varepsilon]=\varepsilon^{-2} \sum_{n=0}^{\infty} \varepsilon^{n} \mathbf{L}^{n} \quad \text { and } \quad \mathbf{B}[\varepsilon]=\varepsilon^{-1} \mathbf{B}^{0}+\mathbf{B}^{1},
$$

whose coefficients are intrinsic operators on $\Gamma$, which are completely determined by the shape of $\Gamma$ and the material parameters of the conducting sheet. We denote by $L_{\alpha}^{n}$ and $B_{\alpha}^{n}$ the surface components of $\mathbf{L}^{n}$ and $\mathbf{B}^{n}$. With the summation convention of repeated two dimensional indices (represented by greek letters), there holds [4, App. A.1, Eq. (A.10)]

$$
L_{\alpha}^{0}(\mathfrak{E})=-\partial_{3}^{2} \mathfrak{E}_{\alpha}+\gamma^{2} \mathfrak{E}_{\alpha} \text { and } L_{\alpha}^{1}(\mathfrak{E})=-2 b_{\alpha}^{\beta} \partial_{3} \mathfrak{E}_{\beta}+\partial_{3} D_{\alpha} \mathfrak{e}+b_{\beta}^{\beta} \partial_{3} \mathfrak{E}_{\alpha},
$$

(we recall that $\gamma$ is defined in (3.5), so that $\left(\kappa_{o}^{\varepsilon}\right)^{2}=-\varepsilon^{-2} \gamma^{2}+\omega^{2} \mu_{o} \epsilon_{o}$ ) and [4, App. A.1, Eq. (A.28)]

$$
B_{\alpha}^{0}(\mathfrak{E})=\partial_{3} \mathfrak{E}_{\alpha} \quad \text { and } \quad B_{\alpha}^{1}(\mathfrak{E})=-D_{\alpha} \mathfrak{e}
$$


Here, $\partial_{3}$ is the partial derivative with respect to $Y_{3}$. We denote by $L_{3}^{n}$ the transverse components of $\mathbf{L}^{n}$. There holds [4, App. A.1, Eq. (A.12)]

$$
L_{3}^{0}(\mathfrak{E})=\gamma^{2} \mathfrak{e} \quad \text { and } \quad L_{3}^{1}(\mathfrak{E})=\gamma_{\alpha}^{\alpha}\left(\partial_{3} \mathfrak{E}\right)+b_{\beta}^{\beta} \partial_{3} \mathfrak{e}
$$

where $\gamma_{\alpha \beta}(\mathfrak{E})=\frac{1}{2}\left(D_{\alpha} \mathfrak{E}_{\beta}+D_{\beta} \mathfrak{E}_{\alpha}\right)-b_{\alpha \beta} \mathfrak{e}$ is the change of metric tensor and $\gamma_{\alpha}^{\alpha}=$ $a^{\alpha \beta} \gamma_{\alpha \beta}$.

5.2. Equations for the coefficients of the electric field. Writing the partial differential equation (5.1b) in the thin conductor $\Omega_{o}^{\varepsilon}$ and the Neumann continuity condition on $\Gamma_{ \pm}^{\varepsilon}$ in the scaled local coordinate system we find that the profiles $\mathfrak{E}_{j}$ and the terms $\mathbf{E}_{j}^{ \pm}$of the electric field (see (2.2)) satisfy the following system (with $\left.I=\left(-\frac{1}{2}, \frac{1}{2}\right)\right)$

$$
\begin{aligned}
& \mathbf{L}[\varepsilon] \sum_{j \geqslant 0} \varepsilon^{j} \mathfrak{E}_{j}\left(y_{\alpha}, Y_{3}\right)=0, \quad \text { in } \Gamma \times I, \\
& \mathbf{B}[\varepsilon] \sum_{j \geqslant 0} \varepsilon^{j} \mathfrak{E}_{j}\left(y_{\alpha}, \pm \frac{1}{2}\right)=\frac{\mu_{o}}{\mu_{ \pm}} \sum_{j \geqslant 0} \varepsilon^{j} \operatorname{curl} \mathbf{E}_{j}^{ \pm} \times \mathbf{n}, \quad \text { on } \quad \Gamma_{ \pm}^{\varepsilon}
\end{aligned}
$$

It is not very convenient that the terms $\mathbf{E}_{j}^{ \pm}$or its derivatives on the right hand side of (5.6b) are evaluated on $\Gamma_{ \pm}^{\varepsilon}$ which moves with $\varepsilon$. However, as the expansion (2.2) of $\mathbf{E}^{\varepsilon}$ is assumed to be valid for any small $\varepsilon>0$, the terms $\mathbf{E}_{j}^{ \pm}$are defined in $\Omega_{ \pm}^{\varepsilon}$ for any $\varepsilon>0$, and, hence, in $\Omega_{ \pm}$. As we have assumed that the thin conductor, and so its mid-surface $\Gamma$, are smooth, that $\mu_{ \pm}, \epsilon_{ \pm}$and $\sigma_{ \pm}$are constants, and that the current $\mathbf{j}$ is zero close to $\Gamma$ it makes sense to accept that the vector fields $\mathbf{E}_{j}^{ \pm}$are regular in a neighbourhood of $\Gamma$. This can be justified using the regularity theory, see e.g. [19, Chap. 4]. Hence, we can use the Taylor expansion and infer for $n \in \mathbb{N}$ that

$$
\begin{gathered}
\mathbf{E}_{n}^{ \pm} \times\left.\mathbf{n}\right|_{h= \pm \frac{\varepsilon}{2}}=\quad \mathbf{E}_{n} \times\left.\mathbf{n}\right|_{0^{ \pm}} \pm \frac{\varepsilon}{2} \partial_{h} \mathbf{E}_{n} \times\left.\mathbf{n}\right|_{0^{ \pm}}+\cdots \\
\operatorname{curl} \mathbf{E}_{n}^{ \pm} \times\left.\mathbf{n}\right|_{h= \pm \frac{\varepsilon}{2}}=\operatorname{curl} \mathbf{E}_{n} \times\left.\mathbf{n}\right|_{0^{ \pm}} \pm \frac{\varepsilon}{2} \partial_{h} \operatorname{curl} \mathbf{E}_{n} \times\left.\mathbf{n}\right|_{0^{ \pm}}+\cdots
\end{gathered}
$$

where $\left.\cdot\right|_{0^{ \pm}}$means the limit for positive or negative $h \rightarrow 0$, respectively. Furthermore, it is convenient to define $\mathbf{E}_{n}$ for $n \in \mathbb{N}$ by $\mathbf{E}_{n}=\mathbf{E}_{n}^{+}$in $\Omega_{+}$, and $\mathbf{E}_{n}=\mathbf{E}_{n}^{-}$in $\Omega_{-}$.

Using the expression of the operator $\mathbf{L}^{0}$, and expanding $\mathbf{E}^{\varepsilon}$ in $\Omega_{ \pm}^{\varepsilon}$, we deduce that, according to the system (5.1) and using (5.6) and (5.7), the profiles $\mathfrak{E}_{n}=\left(\mathfrak{E}_{n}, \mathfrak{e}_{n}\right)$ 
and the terms $\mathbf{E}_{n}$ have to satisfy, for all $n \geq 0$

$$
\begin{array}{rlrl}
L_{3}^{0}\left(\mathfrak{E}_{n}\right)=\gamma^{2} \mathfrak{e}_{n}= & -\sum_{j=1}^{n} L_{3}^{j}\left(\mathfrak{E}_{n-j}\right) & \text { in } \Gamma \times I, \\
L_{\alpha}^{0}\left(\mathfrak{E}_{n}\right)=-\partial_{3}^{2} \mathfrak{E}_{n, \alpha}+\gamma^{2} \mathfrak{E}_{n, \alpha}= & -\sum_{j=1}^{n} L_{\alpha}^{j}\left(\mathfrak{E}_{n-j}\right) & \\
\left.\partial_{3} \mathfrak{E}_{n, \alpha}\right|_{ \pm \frac{1}{2}}= & \left.D_{\alpha} \mathfrak{e}_{n-1}\right|_{ \pm \frac{1}{2}} \\
& +\frac{\mu_{o}}{\mu_{ \pm}} \sum_{j=1}^{n} \frac{1}{( \pm 2)^{j-1}} \partial_{h}^{j-1}\left(\operatorname{curl} \mathbf{E}_{n-j}^{ \pm} \times\left.\mathbf{n}\right|_{0^{ \pm}}\right)_{\alpha} & \text { on } \quad \Gamma, \\
\operatorname{curl} \operatorname{curl} \mathbf{E}_{n}^{ \pm}-\kappa_{ \pm}^{2} \mathbf{E}_{n}^{ \pm}= & \delta_{n}^{0} i \omega \mu_{ \pm} \mathbf{j}_{ \pm} & \text {in } \quad \Gamma \times I, \\
\mathbf{E}_{n}^{ \pm} \times\left.\mathbf{n}\right|_{0^{ \pm}}= & \mathfrak{E}_{n} \times\left.\mathbf{n}\right|_{ \pm \frac{1}{2}}-\sum_{j=1}^{n} \frac{1}{( \pm 2)^{j}} \partial_{h}^{j} \mathbf{E}_{n-j}^{ \pm} \times\left.\mathbf{n}\right|_{0^{ \pm}} & \text {in } \quad \Omega_{ \pm}, \\
\operatorname{curl} \mathbf{E}_{n}^{+} \times \mathbf{n}-i \kappa_{+} \mathbf{n} \times \mathbf{E}_{n}^{+} \times \mathbf{n}=0 & \text { on } \Gamma,
\end{array}
$$

where $\left.\cdot\right|_{ \pm \frac{1}{2}}$ abbreviates the trace on $Y_{3}= \pm \frac{1}{2}$.

5.3. First terms of the asymptotics. In the previous section we have derived the coupled systems for the terms of the asymptotic expansions to any order n. Hence we can determine now the first terms $\mathfrak{E}_{n}=\left(\mathfrak{E}_{n}, \mathfrak{e}_{n}\right)$ and $\mathbf{E}_{n}$ by induction.

The coupled system of order 1 . For $n=0$ in the previous system, we find that $\mathfrak{E}_{0}=\left(\mathfrak{E}_{0}, \mathfrak{e}_{0}\right)$ and $\mathbf{E}_{0}$ satisfy

$$
\begin{aligned}
\gamma^{2} \mathfrak{e}_{0} & =0 & & \text { in } \quad \Gamma \times I, \\
-\partial_{3}^{2} \mathfrak{E}_{0, \alpha}+\gamma^{2} \mathfrak{E}_{0, \alpha} & =0, & & \text { in } \Gamma \times I, \\
\left.\partial_{3} \mathfrak{E}_{0, \alpha}\right|_{ \pm \frac{1}{2}} & =0 & & \text { on } \Gamma, \\
\operatorname{curl} \operatorname{curl} \mathbf{E}_{0}^{ \pm}-\kappa_{ \pm}^{2} \mathbf{E}_{0}^{ \pm} & =i \omega \mu_{ \pm} \mathbf{j}_{ \pm} & & \text {in } \quad \Omega_{ \pm}, \\
\mathbf{E}_{0}^{+} \times\left.\mathbf{n}\right|_{0^{ \pm}} & =\mathfrak{E}_{0} \times\left.\mathbf{n}\right|_{ \pm \frac{1}{2}} & & \text { on } \Gamma, \\
\operatorname{curl} \mathbf{E}_{0}^{+} \times \mathbf{n}-i \kappa_{+} \mathbf{n} \times \mathbf{E}_{0}^{+} \times \mathbf{n} & =0 & & \text { on } \partial \Omega,
\end{aligned}
$$

Obviously, (5.9a) implies with $\gamma \neq 0$ that $\mathfrak{e}_{0}=0$ and in view of (5.9b) and (5.9c) we can assert that $\mathfrak{E}_{0, \alpha}=0$, hence, $\mathfrak{E}_{0}=0$, and the electric fields vanish inside the thin conductor in the limit $\varepsilon \rightarrow 0$. With this the right hand side of (5.9e) is zero as well, i.e., the electric field satisfies the PEC boundary conditions, and we obtain the limit system (3.1)-(3.2) for $\mathbf{E}_{0}^{ \pm}$. 
The coupled system of order 2. Then in the same way as above we find that $\mathfrak{E}_{1}=\left(\mathfrak{E}_{1}, \mathfrak{e}_{1}\right)$ and $\mathbf{E}_{1}$ satisfy

$$
\begin{aligned}
& \gamma^{2} \mathfrak{e}_{1}=-L_{3}^{1}\left(\mathfrak{E}_{0}\right) \quad \text { in } \Gamma \times I, \\
& -\partial_{3}^{2} \mathfrak{E}_{1, \alpha}+\gamma^{2} \mathfrak{E}_{1, \alpha}=-L_{\alpha}^{1}\left(\mathfrak{E}_{0}\right) \quad \text { in } \Gamma \times I, \\
& \left.\partial_{3} \mathfrak{E}_{1, \alpha}\right|_{ \pm \frac{1}{2}}=\left.D_{\alpha} \mathfrak{e}_{0}\right|_{ \pm \frac{1}{2}}+\frac{\mu_{o}}{\mu_{ \pm}}\left(\operatorname{curl} \mathbf{E}_{0}^{ \pm} \times\left.\mathbf{n}\right|_{0^{ \pm}}\right)_{\alpha} \quad \text { on } \quad \Gamma, \quad(5.10 \mathrm{c}) \\
& \operatorname{curl} \operatorname{curl} \mathbf{E}_{1}^{ \pm}-\kappa_{ \pm}^{2} \mathbf{E}_{1}^{ \pm}=0 \quad \text { in } \Omega_{ \pm}, \\
& \mathbf{E}_{1}^{ \pm} \times\left.\mathbf{n}\right|_{0^{ \pm}}=\mathfrak{E}_{1} \times\left.\mathbf{n}\right|_{ \pm \frac{1}{2}} \mp \frac{1}{2} \partial_{h} \mathbf{E}_{0}^{ \pm} \times\left.\mathbf{n}\right|_{0^{ \pm}} \quad \text { on } \quad \Gamma, \\
& \text { on } \partial \Omega \text {. }
\end{aligned}
$$

Perfectly clear, $\mathfrak{E}_{0}=0$ implies $L_{3}^{1}\left(\mathfrak{E}_{0}\right)=0$, and by (5.10a)

$$
\mathfrak{e}_{1}=0
$$

the normal component $\mathfrak{e}_{1}$ of the electric field $\mathfrak{E}_{1}$ of order 1 vanishes just like that of order 0. Similarly, the right hand side of $(5.10 \mathrm{~b})$ is zero just as $D_{\alpha} \mathfrak{e}_{0}$ on the right hand side of (5.10c). Hence, the tangential components are given for $Y_{3} \in\left(-\frac{1}{2}, \frac{1}{2}\right)$ by

$$
\mathfrak{E}_{1}\left(y_{\beta}, Y_{3}\right)=\mathfrak{E}_{1,0}\left(y_{\beta}\right) \cosh \left(\gamma Y_{3}\right)+\mathfrak{E}_{1,1}\left(y_{\beta}\right) \sinh \left(\gamma Y_{3}\right)
$$

with

$$
\mathfrak{E}_{1,0}=\frac{\mu_{o}}{2 \gamma \sinh \left(\frac{\gamma}{2}\right)}\left[\frac{1}{\mu} \operatorname{curl} \mathbf{E}_{0} \times \mathbf{n}\right]_{\Gamma} \quad \text { and } \quad \mathfrak{E}_{1,1}=\frac{\mu_{o}}{\gamma \cosh \left(\frac{\gamma}{2}\right)}\left\{\frac{1}{\mu} \operatorname{curl} \mathbf{E}_{0} \times \mathbf{n}\right\}_{\Gamma} .
$$

This explicit representation inside the thin conductor represents the boundary layer behaviour with a constant relative thickness (since the factor $\gamma=\exp \left(\frac{3 i \pi}{4}\right) \sqrt{\omega \mu_{o} \tilde{\sigma}}$ is independent of $\varepsilon$ ), i.e., the boundary layer thickness scales like the sheet thickness $\varepsilon$.

Now, inserting this explicit representation into the Dirichlet conditions (5.10e), we find that the term $\mathbf{E}_{1}^{-}$solves the following boundary value problem :

$$
\left\{\begin{array}{lll}
\operatorname{curlcurl} \mathbf{E}_{1}^{-}-\kappa_{-}^{2} \mathbf{E}_{1}^{-} & =0 & \text { in } \Omega_{-}, \\
\mathbf{E}_{1}^{-} \times \mathbf{n} & =\mathbf{e}_{1}^{-} \times \mathbf{n} \quad \text { on } \Gamma
\end{array}\right.
$$

and the term $\mathbf{E}_{1}^{+}$satisfies the problem

$$
\left\{\begin{array}{llrl}
\operatorname{curl} \operatorname{curl} \mathbf{E}_{1}^{+}-\kappa_{+}^{2} \mathbf{E}_{1}^{+} & =0 & \text { in } \Omega_{+}, \\
\mathbf{E}_{1}^{+} \times \mathbf{n} & =\mathbf{e}_{1}^{+} \times \mathbf{n} & \text { on } \Gamma, \\
\operatorname{curl} \mathbf{E}_{1}^{+} \times \mathbf{n}-\mathrm{i} \kappa_{+} \mathbf{n} \times \mathbf{E}_{1}^{+} \times \mathbf{n} & =0 & \text { on } \partial \Omega,
\end{array}\right.
$$

with (recalling that $\left.\mathbf{u}_{\mathbf{T}}=\mathbf{n} \times\left(\mathbf{u}_{\mid \Gamma} \times \mathbf{n}\right)\right)$

$\mathbf{e}_{1}^{ \pm}:=\mu_{o}\left(\frac{1}{2 \gamma \tanh \left(\frac{\gamma}{2}\right)}\left[\frac{1}{\mu} \operatorname{curl} \mathbf{E}_{0} \times \mathbf{n}\right]_{\Gamma} \pm \frac{1}{\gamma} \tanh \left(\frac{\gamma}{2}\right)\left\{\frac{1}{\mu} \operatorname{curl} \mathbf{E}_{0} \times \mathbf{n}\right\}_{\Gamma}\right) \mp \frac{1}{2} \partial_{h}\left(\mathbf{E}_{0}^{ \pm}\right)_{\mathbf{T}}$ 
The two first terms in $\mathbf{e}_{1}^{ \pm}$originate from the boundary layer behaviour inside the conductor whereas the last term comes from the Taylor expansion to obtain conditions on the mid-surface $\Gamma$, and not on the two conductor surfaces $\Gamma_{ \pm}^{\varepsilon}$. Exactly, that last term is not considered in the thin layer boundary conditions, see Sec. 3.3.2.

REMARK 5.1. Since the electric field $\mathfrak{E}_{1}$ of order 1 inside the conductor does not vanish, i.e., its tangential components, the normal component $\mathfrak{e}_{2}$ of order 2 is expected to be non-zero. As $L_{3}^{1}\left(\mathfrak{E}_{1}\right)=\gamma_{\alpha}^{\alpha}\left(\partial_{3} \mathfrak{E}_{1}\right)$ this normal component is also a linear combination of $\sinh \left(\gamma Y_{3}\right)$ and $\cosh \left(\gamma Y_{3}\right)$.

6. Acknowledgements. The first and second author gratefully acknowledge the financial support of their research cooperation through the bilateral program "Procope" between the German Academic Exchange Service (DAAD) based on funding of the German Federal Ministry of Education and Research and the French Ministry of Foreign Affairs.

\section{Appendix A. Further notes for deriving the second order condition.}

We have explained in Sec. 2.4 guidelines to derive equivalent conditions from the boundary conditions (2.3c) on $\Gamma$ for the terms of the asymptotic expansions. It is convenient to write the equivalent conditions with Dirichlet and Neumann mean and jump traces.

One may think that the term $\frac{1}{2} \partial_{h}\left(\mathbf{E}_{0}^{ \pm}\right)_{\mathbf{T}}$ in $(5.12 \mathrm{c})$, as it is neither a Dirichlet nor a Neumann trace of $\mathbf{E}_{0}^{ \pm} \in \mathbf{H}\left(\operatorname{curl}, \Omega_{ \pm}\right)$, is not suitable to use in variational formulations or the finite element method. However, we can write according to [24, Prop. 3.36] on $\Gamma$

$$
\partial_{h}\left(\mathbf{E}_{0}^{ \pm}\right)_{\mathbf{T}} \times \mathbf{n}=\left(\operatorname{curl} \mathbf{E}_{0}^{ \pm}\right)_{\mathbf{T}}+\nabla_{\Gamma}\left(\mathbf{E}_{0}^{ \pm} \cdot \mathbf{n}\right) \times \mathbf{n},
$$

and the first term on the right hand side is a multiple of the Neumann trace $\left(\frac{1}{\mu_{ \pm}} \operatorname{curl} \mathbf{E}_{0}^{ \pm}\right)_{\mathbf{T}}$. Using the fact that $\mathbf{E}_{0}^{ \pm}$solves the PDE (3.2a) together with $(\operatorname{curl} \operatorname{curl} \mathbf{u}) \cdot \mathbf{n}=\operatorname{curl} l_{\Gamma}(\operatorname{curl} \mathbf{u})_{\mathbf{T}}$ and $\mathbf{n} \times \operatorname{curl}_{\Gamma}=\nabla_{\Gamma}$ on $\Gamma$ we can rewrite the term $\nabla_{\Gamma}\left(\mathbf{E}_{0}^{ \pm} \cdot \mathbf{n}\right) \times \mathbf{n}$ as

$$
\nabla_{\Gamma}\left(\mathbf{E}_{0}^{ \pm} \cdot \mathbf{n}\right) \times \mathbf{n}=-\frac{\mu_{ \pm}}{\kappa_{ \pm}^{2}} \operatorname{curl}_{\Gamma} \operatorname{curl}_{\Gamma}\left(\frac{1}{\mu_{ \pm}} \operatorname{curl} \mathbf{E}_{0}^{ \pm}\right)_{\boldsymbol{T}} \quad \text { on } \quad \Gamma
$$

Here, we have used that $\left(\operatorname{curl}_{\Gamma} \operatorname{curl}_{\Gamma} \cdot \mathbf{T}\right) \times \mathbf{n} \times \mathbf{n}=-\left(\operatorname{curl}_{\Gamma} \operatorname{curl}_{\Gamma} \cdot\right)_{\mathbf{T}} \operatorname{since}^{\operatorname{curl}_{\Gamma}} \cdot=$ $\left(\nabla_{\Gamma^{\cdot}}\right) \times \mathbf{n}$ is tangent to $\Gamma$. Altogether, we find that

$$
\begin{aligned}
\mathbf{e}_{1}^{ \pm}= & \mu_{o}\left(\frac{1}{2 \gamma \tanh \left(\frac{\gamma}{2}\right)}\left[\frac{1}{\mu} \operatorname{curl} \mathbf{E}_{0} \times \mathbf{n}\right]_{\Gamma} \pm \frac{1}{\gamma} \tanh \left(\frac{\gamma}{2}\right)\left\{\frac{1}{\mu} \operatorname{curl} \mathbf{E}_{0} \times \mathbf{n}\right\}_{\Gamma}\right) \\
& \mp \frac{\mu_{ \pm}}{2}\left(\frac{1}{\mu_{ \pm}} \operatorname{curl} \mathbf{E}_{0}^{ \pm}\right)_{\mathbf{T}} \pm \frac{\mu_{ \pm}}{2 \kappa_{ \pm}^{2}} \operatorname{curl}_{\Gamma} \operatorname{curl}_{\Gamma}\left(\frac{1}{\mu_{ \pm}} \operatorname{curl} \mathbf{E}_{0}^{ \pm}\right)_{\mathbf{T}},
\end{aligned}
$$

which consists of terms depending only on the Neumann traces (from the one or the other side). Finally the Dirichlet conditions for $\mathbf{E}_{1}$ on $\Gamma$ in (5.12) can be written as

$$
\begin{gathered}
{\left[\mathbf{E}_{1} \times \mathbf{n}\right]_{\Gamma}=-\left(2 \frac{\mu_{o}}{\gamma} \tanh \left(\frac{\gamma}{2}\right)-\{\mu\}_{\Gamma}\right)\left\{\frac{1}{\mu}\left(\operatorname{curl} \mathbf{E}_{0}\right)_{\mathbf{T}}\right\}_{\Gamma}+\frac{1}{4}[\mu]_{\Gamma}\left[\frac{1}{\mu}\left(\operatorname{curl} \mathbf{E}_{0}\right)_{\mathbf{T}}\right]_{\Gamma}} \\
-\left\{\frac{\mu}{\kappa^{2}}\right\} \operatorname{curl}_{\Gamma} \operatorname{curl}_{\Gamma}\left\{\left(\frac{1}{\mu} \operatorname{curl} \mathbf{E}_{0}\right)_{\mathbf{T}}\right\}_{\Gamma}-\frac{1}{4}\left[\frac{\mu}{\kappa^{2}}\right]_{\Gamma} \operatorname{curl}_{\Gamma} \operatorname{curl}_{\Gamma}\left[\left(\frac{1}{\mu} \operatorname{curl} \mathbf{E}_{0}\right)_{\mathbf{T}}\right]_{\Gamma},
\end{gathered}
$$


and

$$
\begin{gathered}
\left\{\mathbf{E}_{1} \times \mathbf{n}\right\}_{\Gamma}=-\left(\frac{\mu_{o}}{2 \gamma} \operatorname{coth}\left(\frac{\gamma}{2}\right)-\frac{\{\mu\}}{4}\right)\left[\left(\frac{1}{\mu} \operatorname{curl} \mathbf{E}_{0}\right)_{\mathbf{T}}\right]_{\Gamma}+\frac{1}{4}[\mu]_{\Gamma}\left\{\frac{1}{\mu}\left(\operatorname{curl} \mathbf{E}_{0}\right)_{\mathbf{T}}\right\}_{\Gamma} \\
-\frac{1}{4}\left\{\frac{\mu}{\kappa^{2}}\right\}_{\Gamma} \operatorname{curl}_{\Gamma} \operatorname{curl}_{\Gamma}\left[\left(\frac{1}{\mu} \operatorname{curl} \mathbf{E}_{0}\right)_{\mathbf{T}}\right]_{\Gamma}-\frac{1}{4}\left[\frac{\mu}{\kappa^{2}}\right]_{\Gamma} \operatorname{curl}_{\Gamma} \operatorname{curl}_{\Gamma}\left\{\frac{1}{\mu}\left(\operatorname{curl} \mathbf{E}_{0}\right)_{\mathbf{T}}\right\}_{\Gamma},
\end{gathered}
$$

where we have used the equalities $\{\mathbf{A}\}_{\Gamma}=\{\mu\}_{\Gamma}\left\{\frac{1}{\mu} \mathbf{A}\right\}_{\Gamma}+\frac{1}{4}[\mu]_{\Gamma}\left[\frac{1}{\mu} \mathbf{A}\right]_{\Gamma}$ and $[\mathbf{A}]_{\Gamma}=$ $\{\mu\}_{\Gamma}\left[\frac{1}{\mu} \mathbf{A}\right]_{\Gamma}+[\mu]_{\Gamma}\left\{\frac{1}{\mu} \mathbf{A}\right\}_{\Gamma}$ for any vector field $\mathbf{A}$.

The equivalent conditions (3.3c) of order 2 are then obtained by adding the previous equations multiplied by $\varepsilon$ to the PEC conditions $\left\{\mathbf{E}_{0} \times \mathbf{n}\right\}_{\Gamma}=\left[\mathbf{E}_{0} \times \mathbf{n}\right]_{\Gamma}=0$ (see $(3.1 b)$ and $(3.2 b)$ ) for $\mathbf{E}_{0}$ and by replacing $\mathbf{E}_{0}+\varepsilon \mathbf{E}_{1}$ on the left hand side by the new unknown $\mathbf{E}_{\varepsilon}^{1}$ and by replacing $\varepsilon \mathbf{E}_{0}$ on the right hand side by $\varepsilon \mathbf{E}_{\varepsilon}^{1}$.

\section{REFERENCES}

[1] H Bateman. The Mathematical Analysis of Electrical and Optical Wave-Motion on the basis of Maxwell's Equations. Cambridge University Press, 1915.

[2] O. Biro, K. Preis, K.R. Richter, R. Heller, P. Komarek, and W. Maurer. FEM calculation of eddy current losses and forces in thin conducting sheets of test facilities for fusion reactor components. IEEE Trans. Magn., 28(2):1509-1512, 1992.

[3] D. Braess. Finite Elements: Theory, Fast Solvers, and Applications in Solid Mechanics. Cambridge University Press, 3th edition, 2007.

[4] G. Caloz, M. Dauge, E. Faou, and V. Péron. On the influence of the geometry on skin effect in electromagnetism. Comput. Methods Appl. Mech. Engrg., 200(9-12):1053-1068, 2011.

[5] G. Caloz, M. Dauge, and V. Péron. Uniform estimates for transmission problems with high contrast in heat conduction and electromagnetism. Journal of Mathematical Analysis and Applications, 370(2):555-572, 2010.

[6] G. Cohen and M. Duruflé. Non spurious spectral-like element methods for Maxwell's equations. J. Comput. Math, 25:282-304, 2007.

[7] B. Delourme, H. Haddar, and P. Joly. On the Well-Posedness, Stability And Accuracy Of An Asymptotic Model For Thin Periodic Interfaces In Electromagnetic Scattering Problems. Mathematical Models and Methods in Applied Sciences, 2013.

[8] M. Duruflé, V. Péron, and C. Poignard. Time-harmonic Maxwell equations in biological cellsthe differential form formalism to treat the thin layer. Confluentes Math., 3(2):325-357, 2011.

[9] M. Duruflé, V. Péron, and C. Poignard. Thin layer models for electromagnetism. Commun. Comput. Phys., 16(1):213-238, 2014.

[10] C. Geuzaine, P. Dular, and W. Legros. Dual formulations for the modeling of thin electromagnetic shells using edge elements. IEEE Trans. Magn., 36(4):799-803, 2000.

[11] H. Haddar and Z. Jiang. Axisymmetric eddy current inspection of highly conducting thin layers via asymptotic models. Inverse Problems, 31(11):115005, 2015.

[12] R. Hiptmair. Symmetric coupling for eddy current problems. SIAM J. Numer. Anal., 40(1):4165, 2002.

[13] H. Igarashi, A. Kost, and T. Honma. Impedance boundary condition for vector potentials on thin layers and its application to integral equations. Eur. Phys. J. AP, 1:103-109, 1998.

[14] J.-M. Jin, J. L. Volakis, C.L. Yu, and A.C. Woo. Modeling of resistive sheets in finite element solutions. IEEE Trans. Antennas and Propagation, 40(6):727-731, 1992.

[15] E.F. Knott and T.B.A. Senior. Non-specular radar cross section study. Technical Report 011764-1-T, Univ. Michigan. Radiation Lab., Ann Arbor, MI, USA, Jan 1974.

[16] L. Krähenbühl and D. Muller. Thin layers in electrical engineering. example of shell models in analysing eddy-currents by boundary and finite element methods. IEEE Trans.Mag., 29(2):1450-1455, 1993.

[17] T. Levi-Civita. La teoria elettrodinamica di Hertz di fronte ai fenomeni di induzione. Rend. Lincei (5), 11(2):75-81, 1902. (in Italian).

[18] I.D. Mayergoyz and G. Bedrosian. On calculation of 3-D eddy currents in conducting and magnetic shells. IEEE Transactions on Magnetics, 31(3):1319-1324, 1995. 
[19] William McLean. Strongly Elliptic Systems and Boundary Integral Equations. Cambridge University Press, 2000.

[20] J. McWhirter. Computation of three-dimensional eddy currents in thin conductors. IEEE Trans. Magn., 18(2):456-460, 1982.

[21] P. Monk. Finite element methods for Maxwell's equations. Numerical Mathematics and Scientific Computation. Oxford University Press, New York, 2003.

[22] T. Nakata, N. Takahashi, K. Fujiwara, and Y. Shiraki. 3D magnetic field analysis using special elements. IEEE Trans. Magn., 26(5):2379-2381, 1990.

[23] J-C. Nédélec. Acoustic and electromagnetic equations : integral representations for harmonic problems. Applied mathematical sciences. Springer, New York, 2001.

[24] V. Péron. Modélisation mathématique de phénomènes électromagnétiques dans des matériaux à fort contraste. PhD thesis, Université Rennes 1, France, 2009. http://tel.archivesouvertes.fr/tel-00421736/fr/.

[25] J. Poltz and K. Romanowski. Solution of quasi-stationary field problems by means of magnetic scalar potential. IEEE Trans. Magn., 19(6):2425-2428, 1983.

[26] D. Rodger and N. Atkinson. Finite element method for 3D eddy current flow in thin conducting sheets. IEE Proceedings A, 135(6):369-374, 1988.

[27] K. Schmidt and A. Chernov. Robust families of transmission conditions of high order for thin conducting sheets. Technical Report 1102, Institute for Numerical Simulation, University of Bonn, Feb 2011.

[28] K. Schmidt and A. Chernov. A unified analysis of transmission conditions for thin conducting sheets in the time-harmonic eddy current model. SIAM J. Appl. Math., 73(6):1980-2003, 2013.

[29] K. Schmidt and A. Chernov. Robust transmission conditions of high order for thin conducting sheets in two dimensions. IEEE Trans. Magn., 50(2):41-44, 2014.

[30] K. Schmidt and R. Hiptmair. Asymptotic boundary element methods for thin conducting sheets. Discrete Contin. Dyn. Syst. Ser. S, 8(3):619-647, 2015.

[31] K. Schmidt and S. Tordeux. Asymptotic modelling of conductive thin sheets. Z. Angew. Math. Phys., 61(4):603-626, Aug 2010.

[32] K. Schmidt and S. Tordeux. High order transmission conditions for thin conductive sheets in magneto-quasistatics. ESAIM: Math. Model. Numer. Anal., 45(6):1115-1140, Nov 2011.

[33] T. Senior. Approximate boundary conditions. IEEE Trans. Antennas and Propagation, 29(5):826-829, 1981.

[34] O.V. Tozoni and I.D. Mayergoyz. Analysis of three-dimensional electromagnetic fields. Technika, Kiev, 1974. in russian.

[35] Barbara I. Wohlmuth. A mortar finite element method using dual spaces for the Lagrange multiplier. SIAM J. Numer. Anal., 38(3):989-1012, 2001. 